\title{
Functional diversity and evolution of the Drosophila sperm proteome
}

\author{
Martin D. Garlovsky ${ }^{\mathrm{a}, \mathbb{},}$, Jessica Sandler ${ }^{\mathrm{b}}$, and Timothy L. Karr ${ }^{\mathrm{b}, \mathrm{c}, \mathbb{}, \mathrm{d}}$ \\ a'Department of Applied Zoology, Faculty of Biology, Technische Universität Dresden, Dresden 01069, Germany \\ ${ }^{\mathrm{b}}$ Biosciences Mass Spectrometry Core Research Facility, Knowledge Enterprise, Arizona State University, USA \\ ${ }^{c}$ Neurodegenerative Disease Research Center, The Biodesign Institute, Arizona State University, USA
}

Given the central role fertilization plays in the health and fitness of sexually reproducing organisms and the well-known evolutionary consequences of sexual selection and sperm competition, knowledge gained by a deeper understanding of sperm 5 (and associated reproductive tissues) proteomes has proven critical to the field's advancement. Due to their extraordinary complexity, proteome depth-of-coverage is dependent on advancements in technology and related bioinformatics, both of which have made significant advancements in the decade since the last

10 Drosophila sperm proteome was published. Here we provide an updated version of the Drosophila melanogaster sperm proteome (DmSP3) using improved separation and detection methods and an updated genome annotation. We identified 2563 proteins, with label-free quantitation (LFQ) for 2125 proteins. Combined 15 with previous versions of the sperm proteome, the DmSP3 contains a total of 3176 proteins. The top 20 most abundant proteins contained the structural elements $\alpha$ - and $\beta$-tubulins and sperm leucyl-aminopeptidases (S-Laps). Both gene content and protein abundance were significantly reduced on the $X$ chromo-

20 some, a finding consistent with prior genomic studies of the $\mathbf{X}$ chromosome gene content and evolution. We identified 9 of the 16 Y-linked proteins, including known testis-specific male fertility factors. LFQ measured significant levels for $75 / 83$ ribosomal proteins (RPs) we identified, including a number of core

25 constituents. The role of this unique subset of RPs in sperm is unknown. Surprisingly, our expanded sperm proteome also identified 122 seminal fluid proteins (Sfps), proteins found predominantly in the accessory glands. The possibility of tissue contamination from seminal vesicle or other reproductive tis30 sues was addressed using concentrated salt and detergent treatments. Salt treatment had little effect on sperm proteome composition suggesting only minor contamination during sperm isolation while a significant fraction of Sfps remained associated with sperm following detergent treatment suggesting Sfps may 35 arise within, and have additional functions, in sperm per se.

Keywords: Spermatozoa | seminal fluid proteins | ribosomes | meiotic sex chromosome inactivation | fertility | evolution | discovery proteomics | human disease | OMIM | Drosophila

Abbreviations: AUC, area-under-the-curve; BP, biological processes; CC, cel40 lular components; CDS, coding sequences; CID, collision-induced fragmentation; DAVID, database for visualisation and integrated discovery; DmSP, Drosophila melanogaster sperm proteome; FBgn, FlyBase gene number; FDR, false discovery rate; FPKM, fragments per kilobase of transcript per million mapped reads; GO, Gene ontology; LFQ, Label-free quantitation; MF,

45 molecular functions; MIPS, monoisotopic peak determination; OMIM, Online Mendelian Inheritance in Man; PAML, phylogenetic analysis by maximum likelihood; RPs, ribosomal proteins; S-Laps, sperm leucyl-aminopeptidases; Sfp, Seminal fluid protein; TEAB, triethylammonium bicarbonate

Correspondence: martin.garlovsky@tu-dresden.de; tkarr@asu.edu

\section{Introduction}

Spermatozoa form, function and evolution is determined in large measure by its proteome (1). High throughput proteomics using liquid-chromatography tandem massspectrometry (LC-MS) has now been used to characterize the composition of the sperm proteome in a wide range of animals (1-3). These studies have revealed several common features of sperm as expected for an ancient cell type with a highly conserved function despite exhibiting exceptional morphological diversity across the tree of life (4-6). For instance, across taxa, sperm show enrichment of metabolic processes, mitochondria, axoneme, microtubules and cytoskeletal components $(2,3,7,8)$.

Recent advances in LC-MS technology, particularly in data acquisition time and improved liquid chromatographic systems, provide enhanced proteome coverage of complex cell and tissue types $(9,10)$. These advances accordingly allow routine and accurate quantitation of both label- and label-free methodologies, an essential element for comparative studies of sperm composition and function $(11,12)$. Additionally, these advances permit direct injection of sample peptides without the need for pre-fractionation using polyacrylamide gel electrophoresis thus avoiding sample loss. Accordingly, in the current study we re-interrogated the Drosophila melanogaster sperm proteome using direct solubilization of sperm followed by on-line fractionation of tryptic peptides and report on a significant increase in both proteome size and content.

D. melanogaster with its excellent genome annotation provides a powerful genetic and functional genomics model system to understand reproduction and fertility (e.g., (13)). Our previous efforts identified over 1,000 D. melanogaster sperm proteins with prior versions designated DmSP1 (7) and DmSP2 (14). The DmSP3 described in this study significantly increases coverage and refinement of the $D$. melanogaster sperm proteome, from the 1108 sperm proteins identified in the combined DmSP1 and DmSP2 (14) to more than 3000 proteins in the DmSP3 (Table 1). Table 1 highlights our extended knowledge base not only in terms of absolute numbers of sperm proteins, but also discovery of new protein groups including the surprising findings of substantial numbers of seminal fluid proteins and ribosomal proteins in the DmSP3. We use the increased proteome coverage and quantitative LFQ information in the DmSP3 to provide a detailed analysis of relative abundance of sperm proteins for the 
ime, and re-examine the evolutionary dynamics, gene age, and chromosomal distribution of proteins in the DmSP3. The analyses provide stronger support for previous claims and in particular cements the subjective prior findings supporting the meiotic sex-chromosome inactivation model for male-specific gene and X chromosome evolution (15-18).

\section{Methods}

A. Fly stocks and sample preparation. We used laboratory wild-type strain Oregon-R D. melanogaster males, aged 5-7 days. All dissections tissue removal and sperm isolaphosphate buffered saline (PBS) with or without protease inhibitors (HALT, Thermo Fisher). We anaesthetized flies and removed reproductive tracts with forceps under a stereo dissecting microscope as previously described (14). Briefly, vesicles) were prepared separately over the course of no more than one hour by first removing the seminal vesicles from each male reproductive tract (containing testes, seminal vesicles, and accessory glands) into a fresh drop of PBS. Sperm crocentrifuge tube containing PBS (on ice). Sperm were then pelleted at $15,000 \mathrm{rpm}$ for 15 minutes at $4^{\circ} \mathrm{C}$, washed $3 \mathrm{X}$ with PBS and immediately solubilized in 25 microliters of 5\% SDS/50mM TEAB containing 50mM dithiothreitol. Solubiand spun again at $15,000 \mathrm{rpm}$ for 15 minutes at $20^{\circ} \mathrm{C}$. No visible pellets were observed and the supernatants removed and stored at $-20^{\circ} \mathrm{C}$ or immediately processed as described below.

Solubilized sperm proteins were quantified using EZQ lated (Pierce) using 40mM final concentration freshly prepared iodoacetamide for 30 minutes in the dark at room temperature. Samples were processed using the Protifi S-trap Micro Columns and instructions were given via the S-trap Ultra ified by addition of $12 \%$ phosphoric acid to a final concentration of $\sim 1.2 \%$ phosphoric acid. Proteins were digested by addition of $2.0 \mu \mathrm{g}$ of porcine trypsin (MS grade, Pierce) and incubated at $30^{\circ} \mathrm{C}$ for 2 hours. S-trap buffer ( $90 \%$ methanol, sample volume. Acidified sample and the S-trap buffer was filtered through columns. Columns were washed $3 \mathrm{X}$ with $\mathrm{S}-$ trap buffer. An additional $0.5 \mu \mathrm{g}$ of trypsin and $25 \mu \mathrm{L}$ of 50 $\mathrm{mM}$ TEAB was added to the top of each column and incucolumns using three elution buffers: $50 \mathrm{mM}$ TEAB, $0.2 \%$ formic acid in water, and $50 \%$ acetonitrile $/ 50 \%$ water $+0.2 \%$ formic acid. Samples were dried down via speed vac and resuspended in $20-30 \mu \mathrm{L}$ of $0.1 \%$ formic acid.

\section{B. Liquid-chromatography tandem trometry. All LC-MS analyses were} the Biosciences Mass Spectrometry

mass-specperformed at State University. All data-dependent mass spectra were collected in positive mode using an Orbitrap Fusion Lumos mass spectrometer (Thermo Scientific) coupled with an UltiMate 3000 UHPLC (Thermo Scientific). One $\mu \mathrm{L}$ of peptides were fractionated using an Easy-Spray LC column $(50 \mathrm{~cm} \times 75 \mu \mathrm{m}$ ID, PepMap C18, $2 \mu \mathrm{m}$ particles, $100 \AA$ pore size, Thermo Scientific) equipped with an upstream $300 \mathrm{um} \times 5 \mathrm{~mm}$ trap column. Electrospray potential was set to $1.6 \mathrm{kV}$ and the ion transfer tube temperature to $300^{\circ} \mathrm{C}$. The mass spectra were collected using the "Universal" method optimized for peptide analysis provided by Thermo Scientific. Full MS scans $(375-1500 \mathrm{~m} / \mathrm{z}$ range) were acquired in profile mode with the Orbitrap set to a resolution of 120,000 (at $200 \mathrm{~m} / \mathrm{z}$ ), cycle time set to 3 seconds and mass range set to "Normal". The RF lens was set to $30 \%$ and the AGC set to "Standard". Maximum ion accumulation time was set to "Auto". Monoisotopic peak determination (MIPS) was set to "peptide" and included charge states 2-7. Dynamic exclusion was set to $60 \mathrm{~s}$ with a mass tolerance of $10 \mathrm{ppm}$ and the intensity threshold set to 5.0e3. MS/MS spectra were acquired in a centroid mode using quadrupole isolation window set to $1.6(\mathrm{~m} / \mathrm{z})$. Collision-induced fragmentation (CID) energy was set to $35 \%$ with an activation time of 10 milliseconds. Peptides were eluted during a 240-minute gradient at a flow rate of $0.250 \mathrm{uL} / \mathrm{min}$ containing $2-80 \%$ acetonitrile/water as follows: $0-3$ minutes at $2 \%, 3-75$ minutes $2-15 \%, 75-180$ minutes at $15-30 \%, 180-220$ minutes at $30-35 \%, 220-225$ minutes at $35-80 \% 225-230$ at $80 \%$ and $230-240$ at $80-5 \%$.

C. Label-free quantification (LFQ). We analysed raw files searched against the Uniprot (www.uniprot.org) $D$. melanogaster database (Dmel_UP000000803.fasta) using Proteome Discover 2.4 (Thermo Scientific). Raw files were searched using SequestHT that included Trypsin as enzyme, maximum missed cleavage site $3, \mathrm{~min} / \mathrm{max}$ peptide length $6 / 144$, and precursor ion (MS1) mass tolerance set to $20 \mathrm{ppm}$ and fragment mass tolerance set to $0.5 \mathrm{Da}$ and a minimum of 1 peptide identified. Carbamidomethyl (C) was specified as fixed modification, and dynamic modifications set to Aceytl and Met-loss at the N-terminus, and oxidation of Met. A concatenated target/decoy strategy and a false-discovery rate (FDR) set to $1.0 \%$ was calculated using Percolator (19). The data was imported into Proteome Discoverer 2.4, and accurate mass and retention time of detected ions (features) using Minora Feature Detector algorithm. The identified Minora features were then used to determine area-under-the-curve (AUC) of the selected ion chromatograms of the aligned features across all runs and relative abundances calculated.

D. Gene ontology enrichment. We performed gene ontology (GO) enrichment network analyses using the website version of DAVID (v6.8) (20) and Cytoscape (v3.9.0) (21). We used the ClueGO plugin v2.5.8 (22) for Cytoscape to generate enriched GO categories using a right-sided hypergeometric test and $p$-values, adjusted using BenjaminiHochberg for multiple testing correction, reported. We performed network comparisons between the DmSP2 and DmSP3 using ClueGO. Gene lists were uploaded to DAVID 
Table 1. History of the Drosophila melanogaster sperm proteome (DmSP). DmSP1: (7); DmSP2: (14); DmSP3: this study. The DmSP2 combined the 341 proteins identified in the DmSP1 with the 956 proteins identified in the DmSP2. Likewise, the DmSP3 reported here represents the combined total of all proteins identified in the DmSP2 $(\mathrm{n}=$ 1108) with the 2563 proteins identified across all experiments in the current study. Numbers in parentheses denote number of newly identified proteins. ${ }^{*}$ Under $=$ significant gene underrepresentation compared to expected value (see Methods); ns = not significant.

\begin{tabular}{lccc} 
& DmSP1 & DmSP2 & DmSP3 \\
\hline Methods/Technology & LC-MS ${ }^{2} /$ Maldi & SDS-PAGE & Cell digest \\
Machine & Thermo LCQ & LTQ Orbitrap & Orbitrap Fusion Lumos \\
Proteins identified & 341 & $1108(+767)$ & $3176(+2068)$ \\
X-linked* & Under & ns & Under \\
Y-linked & - & 4 & $9(+5)$ \\
Sfps & CG2918 & $11(+10)$ & $122(+111)$ \\
Ribosomal proteins & - & 9 & $83(+74)$ \\
\hline
\end{tabular}

(https://david.ncifcrf.gov/tools.jsp) and functional outputs for all three GO categories (BP, CC, MF) and associated statistical values were saved in Excel spreadsheets. Enriched GO categories with FDR values below $1 \%$ are reported. Specific parameters details are found in the figure legends.

E. Evolutionary rates. We calculated the rate of nonsynonymous $(\mathrm{dN})$ to synonymous $(\mathrm{dS})$ nucleotide substitutions (dN/dS) for D. melanogaster genes using an existing pipeline (23). We downloaded amino acid sequences and ${ }_{215}$ coding sequences (CDS) for D. melanogaster (BDGP6.32), and CDS files for D. sechellia (dsec_r1.3), D. simulans (ASM75419v3), and D. yakuba (dyak_caf1) from Ensembl (24). For each species, we identified the longest isoform of each gene and identified orthologs using reciprocal BLASTn

220 (25), with a minimum $30 \%$ identity and 1x10-10 E-value cut-off. We identified reciprocal 1:1 orthologs between all four species by the highest BLAST score and identified open reading frames using BLASTx. We then aligned orthologs using PRANK (26) and masked poorly aligned reads with synonymous substitution threshold $=7$, and window size $=$ 15. We retained 11715 orthologs for analysis after filtering poorly aligned orthologs and those with sequence length $<30 \mathrm{bp}$. We calculated one-ratio estimates (model 0) with

230 an unrooted phylogeny: ((D. simulans, D. sechellia $), D$. melanogaster, D. yakuba), using the CODEML package in PAML (28), and filtered orthologs with a branch specific dS $\geq 2$ or where $\mathrm{S} * \mathrm{~d} S \leq 1$ to avoid mutational saturation. In total we retained dN/dS estimates for 11417 genes after filtering, including 2571 (80.9\%) proteins in the DmSP3. We tested for differences in evolutionary rates between independent sets of genes using Mann-Whitney U tests.

\section{F. Experimental design and statistical rationale. We} designed experiments to (i) maximize proteome coverage,

240 (ii) measure using label-free quantitation the relative abundance of individual proteins in the proteome and (iii) examine sample purity by measuring the magnitude of adventitious protein binding and contamination in our samples. We performed three independent experiments using three treat-

ments of purified sperm samples as described in Methods. In experiment one, we collected 3 biological replicates of sperm in PBS only. In experiment two sperm were collected in either PBS and Halt protease inhibitor ("Halt" treatment), PBS only ("NoHalt" treatment) or PBS containing 0.1\% Triton X100 without protease inhibitor ("PBST" treatment). In experiment three we collected 4 biological replicates of sperm prepared using either PBS ("PBS" treatment) or $2.5 \mathrm{M} \mathrm{NaCl}$ ("Salt" treatment).

We applied strict thresholds for peptide and protein identification by setting a false-discovery rate (FDR) threshold at $1.0 \%$, calculated using a reverse-concatenated target/decoy strategy in Percolator. To test for differences in abundance between treatments, LFQ ion intensities, calculated using the Minora feature detector in Proteome Discoverer to determine area-under-the-curve (AUC) and summed technical replicates prior to analysis precursor intensities, were fit to protein-wise negative binomial generalized linear models. For experiment two, investigating the effect of detergent treatment, during preliminary analysis we performed pairwise analysis between all three treatments which revealed 16 proteins that showed differential abundance between controls (Halt vs. NoHalt) (Fig. S1). We subsequently performed differential abundance analysis comparing the PBST treatment to the average of both controls (Halt and NoHalt), excluding these 16 proteins (see supplementary analysis). To rank order protein abundances, we calculated a grand mean for each protein, excluding the PBST treatment samples which, as expected, showed substantial differences compared to other samples (see Results).

G. Statistical analysis. We performed all statistical analysis in R v4.03 (29). All code and analyses are available via GitHub.

To test for non-random distribution of sperm proteins across the polytene chromosomes we downloaded the chromosomal location for all genes in the genome from FlyBase.org (30) and calculated the total numbers of genes on each chromosome. We then summed the observed number of genes found in the sperm proteome on each chromosome, and calculated the expected number based on the total number of sperm proteins identified. We calculated $\chi^{2}$ statistics for each chromosome and the associated $p$-values with one degree of freedom and used the Benjamini-Hochberg procedure to correct for multiple testing. We excluded analysis of the $\mathrm{Y}$ chromosome due to the small number of protein coding genes. To test for non-random distribution of sperm genes across ages classes, we downloaded gene age information from http://gentree.ioz.ac.cn/download.php (31) and
250 
grouped as; ancestral (class 0; common to the Drosophila genus; $\mathrm{n}=12013$ ), subgenus Sophophora (classes $1+2$; $\mathrm{n}$ $=416$ ), melanogaster group (class $3 ; \mathrm{n}=200$ ), melanogaster subgroup (class $4 ; n=334$ ), or recent (classes $5+6 ; n=120$ ). We tested if sperm genes were randomly distributed across age classes compared to the rest of the genome as above, calculating the observed number of genes in each age class across the genome and among sperm proteins and calculating $\chi^{2}$ statistics comparing the observed vs. expected number of genes in each age class, using the Benjamini-Hochberg procedure to correct for multiple testing.

To test for differences in abundance between ribosogroups of X-linked- Y-linked- or autosomal- proteins, or between 'high confidence' seminal fluid proteins (Sfps), 'low confidence/transferred' Sfps, or remaining sperm proteins, we calculated the grand mean abundance across all three exteins identified by two or more unique peptides and found in at least 3 biological replicates in at least 1 treatment group (where applicable). We performed Kruskal-Wallace ranksum tests followed by pairwise Wilcoxon rank-sum tests corfor multiple testing using the Benjamini-Hochberg procedure. For experiments two and three we performed differential abundance analyses using edgeR (32). For experiment two we filtered proteins with values in 7 out of 9 biological replicates. For experiment three we filtered to include proteins identified in at least 5 replicates (i.e., in at least 3 out of 4 biological replicates of one treatment).

\section{Results}

H. Overview of the DmSP3. In the current study we identified 2563 proteins across our three experiments (Fig. S2), more unique peptides in a single experiment $(n=1412)$ or in two or more replicates across any experiment $(n=1867)$. Relative protein abundances of 2125 proteins $(81.2 \%)$ were measured by LFQ. As expected from our previous study (33), Laps) were among the most abundant (Table 2). Also present were proteins of unexpected sperm prevalence including ocnus and janus B, a pair of duplicated gene products encoding a testis-specific phosphohistidine phosphatase (34), numerteins (RPs). Overall, we found highly consistent estimates of protein abundances between experiments. Protein abundances were strongly correlated between experiments (Pearson's correlation $=0.86-0.89$, all $p<0.001 ;$ Fig. S3) and median coefficients of variation for each experiment ranged from $0.018-0.054$. We performed analyses using the entire DmSP3 ( $\mathrm{n}=3176$; Table S1), combining the 2563 proteins identified in the current study with the 1108 proteins identified in the DmSP2 $(7,14)$ (Fig. 1a).

I. Gene Ontology and network analyses. The DmSP3 is considerably larger than the DmSP2 (Fig. 1a) and GO analysis identified 24 significantly enriched BP categories (Fig. 2a;
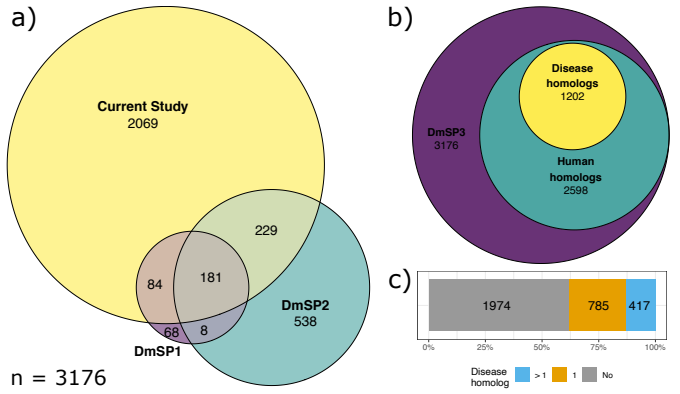

Fig. 1. Proteins identified in the D. melanogaster sperm proteome (DmSP). a) Overlap between DmSP1, DmSP2 and the current study, together making up the DmSP3 $(n=3176)$. b) Number of $D$. melanogaster genes found in the DmSP3 with human homologs and disease associated phenotypes from the Online Mendilian Inheritance in Man database (OMIM.org). c) Number of $D$. melanogaster sperm protein genes with none (grey), one (orange), or more than one (blue) associated disease phenotype.

Table S2). As expected, major categories included processes involved in energy transduction (e.g., oxidation-reduction, glycolysis, TCA cycle) and reproduction. Other spermspecific functions included terms related to microtubule and cilium movement. Surprisingly, the GO term "translation" was a prominent member in this analysis containing $78 \mathrm{cy}-$ tosolic and mitochondrial RPs. To further explore the GO category representation in the DmSP2 and DmSP3, we generated a heat map between the two proteomes in Cytoscape using ClueGO (Fig. 2b). Similar to our previous analysis of the DmSP1 and DmSP2 (14), most of the categories were equal or nearly equal in their shared properties with the one obvious exception being the aforementioned translation BP category as discussed further below.

J. Human disease homologs. Genes in the DmSP3 are highly conserved, with $81.8 \%$ (2598/3176) of genes having

Table 2. Most abundant proteins in the DmSP3. Top 20 most abundant proteins in the DmSP3 by LFQ (rank ordered).

\begin{tabular}{llc} 
FBgn & Name & Chrom. \\
\hline FBgn0003884 & $\alpha$-Tubulin at 84B & 3R \\
FBgn0003889 & $\beta$-Tubulin at 85D & 3R \\
FBgn0259795 & loopin-1 & 2R \\
FBgn0003885 & $\alpha$-Tubulin at 84D & 3R \\
FBgn0033868 & Sperm-Leucylaminopeptidase 7 & 2R \\
FBgn0035915 & Sperm-Leucylaminopeptidase 1 & 3L \\
FBgn0052064 & Sperm-Leucylaminopeptidase 4 & 3L \\
FBgn0045770 & Sperm-Leucylaminopeptidase 3 & 3L \\
FBgn0039071 & big bubble 8 & 3R \\
FBgn0034132 & Sperm-Leucylaminopeptidase 8 & 2R \\
FBgn0041102 & ocnus & 3R \\
FBgn0031545 & CG3213 & 2L \\
FBgn0037862 & Mitochondrial aconitase 2 & 3R \\
FBgn0038373 & CG4546 & 3R \\
FBgn0002865 & Male-specific RNA 98Ca & 3R \\
FBgn0035240 & CG33791 & 3L \\
FBgn0025111 & Adenine nucleotide translocase 2 & X \\
FBgn0069354 & Porin2 & 2L \\
FBgn0052351 & Sperm-Leucylaminopeptidase 2 & 3L \\
FBgn0012036 & Aldehyde dehydrogenase & 2L \\
\hline
\end{tabular}


bioRxiv preprint doi: https://doi.org/10.1101/2022.02.14.480191; this version posted February 14, 2022. The copyright holder for this preprint (which was not certified by peer review) is the author/funder, who has granted bioRxiv a license to display the preprint in perpetuity. It is made available under aCC-BY-NC-ND 4.0 International license.

J Human disease homologs

a)

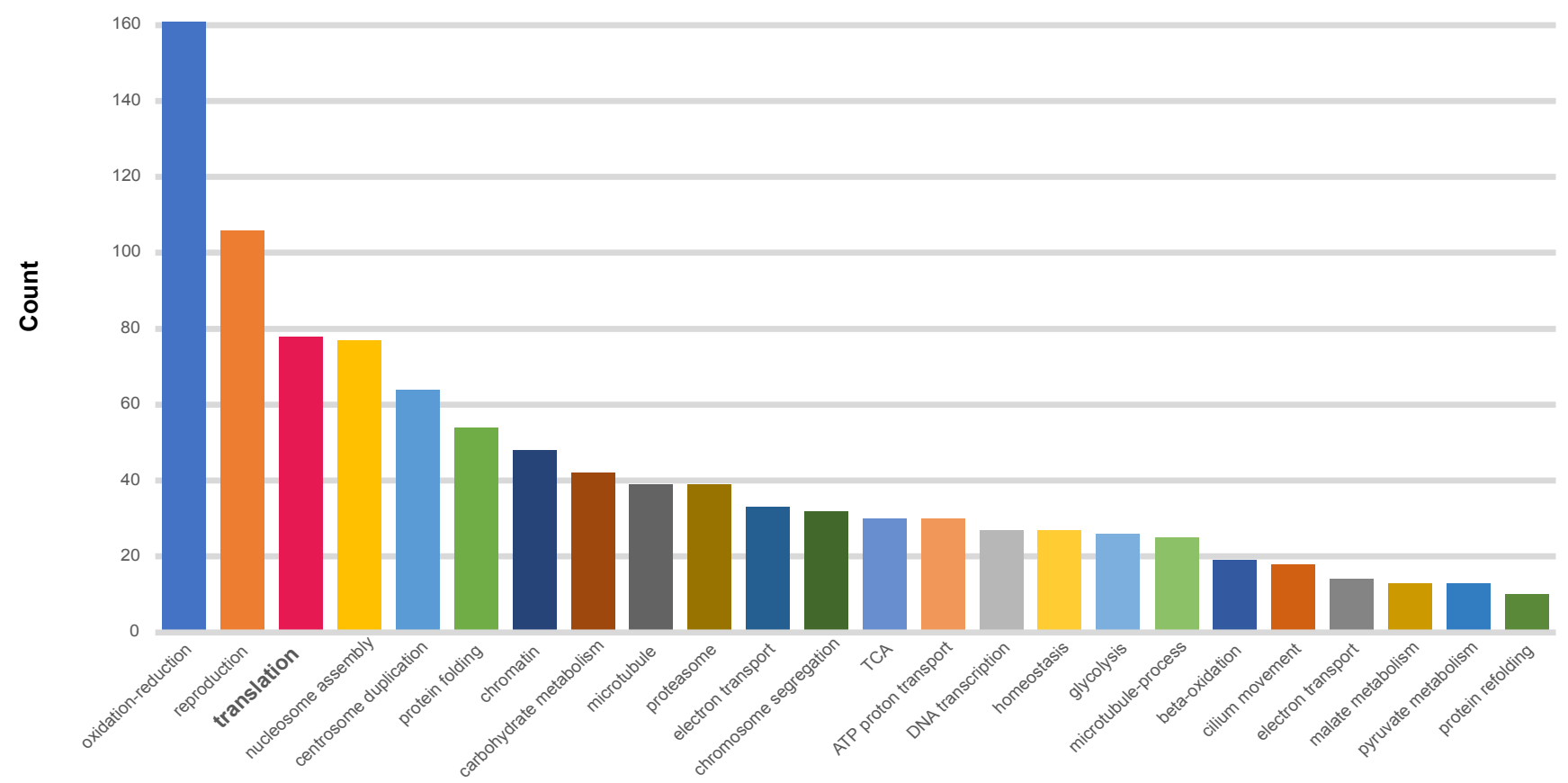

b)

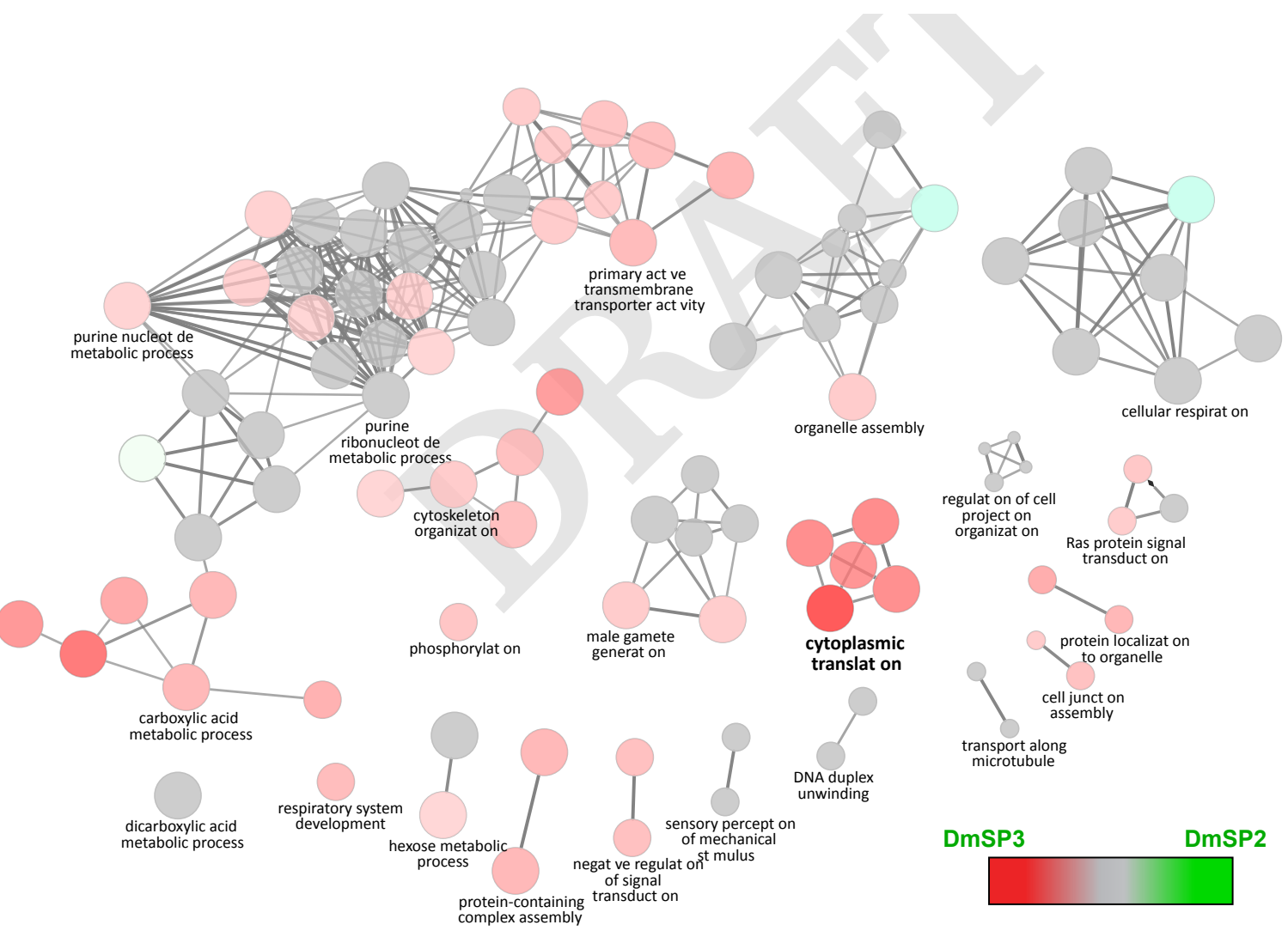

Fig. 2. GO functional network enrichment analysis and comparison of the DmSP2 and DmSP3. (a) Bar graph of the 24 GO Biological Process categories identified in the DmSP3 by DAVID (20). Only functional enrichment groups with Benjamini-Hochberg corrected $p$-values $<0.01$ and passing a $1 \%$ FDR threshold are shown. Note: some GO terms have been combined for clarity; see Table S2 for complete list of GO terms. (b) GO Biological Process network comparison between the DmSP3 (3176 proteins) and DmSP2 (1108 proteins) using the ClueGO plugin for Cytoscape. Color-coded nodes within the network depict the degree of relative compositional enrichment of each dataset. The network is comprised of 22 groups (each comprised of at least 30 genes associated with a common GO functional term) containing a total of 1431 proteins. Node compositional enrichment for proteins identified in the current study (highlighted in red) when node composition bias exceeds $60 \%$ while grey nodes indicate equal representation. Bold letters indicate one highly enriched category of proteins involved in cytoplasmic translation.

human homologs, compared to $48 \%$ of all Drosophila genes 365
(35). Fully $37.8 \%$ (1202/3176) of DmSP3 genes have a ho- molog in humans associated with a known disease or syndrome in a search against the Online Mendelian Inheritance 
in Man database (OMIM.org; Fig. 1b). Over one third $(34.7 \%$; 417/1202) of disease associated DmSP3 genes have more than one human disease homolog (Fig. 1c). Among the most prevalent disease phenotypes found were susceptibility to autism, primary ciliary dyskinesia, spermatogenic failure, and myofibrillar- and congenital- myopathy (Table 3 ).

K. Ribosomal proteins in the DmSP3. Almost one-half of all D. melanogaster RPs listed in FlyBase.org (83/169; $49.1 \%$, including paralogs) were identified in the DmSP3 (Table S3). We identified the majority of cytoplasmic RPs (76/93; 81.7\%) but only 9.2\% (7/76) of mitochondrial RPs. There was no significant difference in RP abundance compared to the DmSP3 average (Kruskal-Wallis rank sum test, $\chi^{2}=0.063, \mathrm{df}=1, p=0.803$; Fig. 3a), suggesting that RPs identified in the DmSP3 are integral to the sperm proteome and not artefactual.

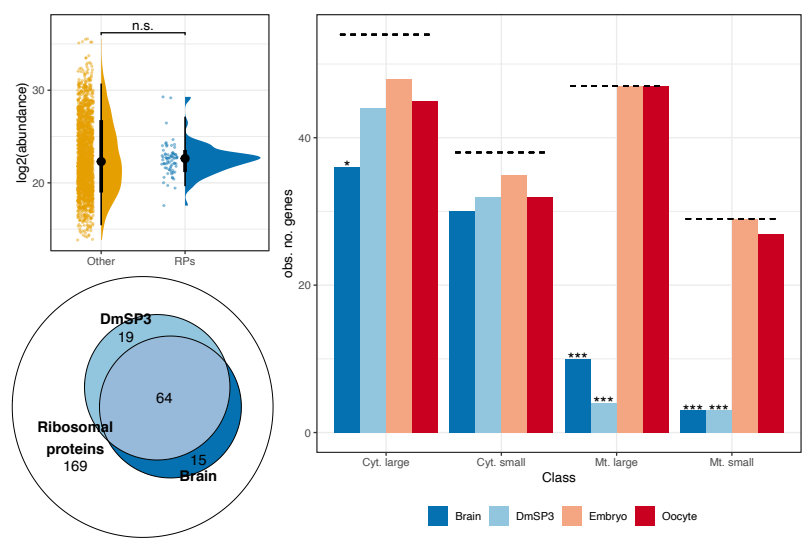

Fig. 3. Ribosomal proteins in the DmSP3. a) Abundance of ribosomal proteins (RPs) identified in the DmSP3 compared to the remaining sperm proteome ('other'). Colored points represent the abundance of individual proteins. Black points show the mean and thick and thin bars represent the $33 \%$, and $66 \%$ confidence intervals, respectively. We compared abundances using a Kruskal-Wallace rank sum test. b) Representation of large and small cytoplasmic and mitochondrial RPs in the brain, DmSP3, embryo, or oocyte. The dashed line represents the total number of RPs in each class and asterisks represent results from comparing the observed to expected number of proteins identified using the $\chi^{2}$ distribution after multiple testing correction. c) Overlap between the total number of RPs identified in the DmSP3 and brain tissue. n.s.; non-significant; ${ }^{*} p<0.05$; ${ }^{* *} p<0.01 ;{ }^{* * *} p<0.001$.

The canonical ribosome contains 80 RPs including 13

paralog pairs in Drosophila (FlyBase.org). Although the significance of paralog heterogeneity for ribosome function is currently unknown, paralog switching of RPs has been observed in gonads and other tissues (36). We therefore compared RP paralogs in the DmSP3 to those previously described in four tissue types including the testis (36). Significant differences were found between all four tissues as only three RP paralogs were observed in the DmSP3 (RpL22-like, $\mathrm{RpS} 14 \mathrm{~b}$ and RpS28b) whereas both RPs and paralog RPs were found in all tissues with the exception of two (Rp10Aa RpS14a; Table S4). Notably, RpL22-like is more abundant in the testis (36), whereas RpL22 was more abundant in the DmSP3. For the remaining paralogs we identified only one member of each pair: the most abundant paralog found in the testis for seven, and the less abundant paralog for two (Table S4). For RpL10Ab and RpS14b only one paralog was identified in both the current study and by Hopes et al. (36), and we did not identify either paralog of RpS10 (RpS10a or $\mathrm{RpS10b}$ ). Together, these results suggest a complex landscape of paralog switching in the gonad during spermatogenesis and highlight distinct differences between sperm-RP and testis-RP populations.

We next compared the representation of RPs found in the DmSP3 to three other recent proteomic studies in D. melanogaster which used Lumos Fusion Orbitrap massspectrometers; embryo (37), unfertilised oocyte (38), and brain (39). All four tissue/cell types identified most cytoplasmic RPs, with a slight underrepresentation of large cytoplasmic subunits in the brain (Fig. 3b). The DmSP3 and brain both showed significant underrepresentation of large and small mitochondrial RPs, whereas oocyte and embryo showed almost complete representation of all ribosomal subunits (Fig. 3b). Significantly more RPs identified in the brain or sperm were shared between tissues $(64 / 98 ; 65.3 \%)$ than expected by chance (Fisher's exact test, $p<0.001$; Fig. 3c).

L. Chromosomal distribution of sperm proteins. Sperm proteins were underrepresented on the $\mathrm{X}-\left(\chi^{2}=12.6, \mathrm{df}=1, p\right.$ $=0.002)$ and $3 \mathrm{~L}-\left(\chi^{2}=11.8, \mathrm{df}=1, p=0.002\right)$ chromosomes (Fig. 4a); a pattern that was previously reported for X-linked genes in the DmSP1 (7) but not replicated in the DmSP2 (14). Protein abundance of X-linked proteins was significantly lower than those on autosomes (Wilcoxon rank-sum test, $p=0.041$ ) or the $\mathrm{Y}$ chromosome (Wilcoxon rank-sum test, $p<0.001$; Fig. $4 \mathrm{~b}$ ). We identified 9 of the 16 known proteins encoded on the $\mathrm{Y}$ chromosome (Table 4). The average abundance of Y-linked sperm proteins was higher than autosomal sperm proteins (Wilcoxon rank-sum test, $p<0.001$ ); 6 within the top $20 \%$ most highly abundant proteins, and all within the top 50\% (Fig. 4b; Table 4).
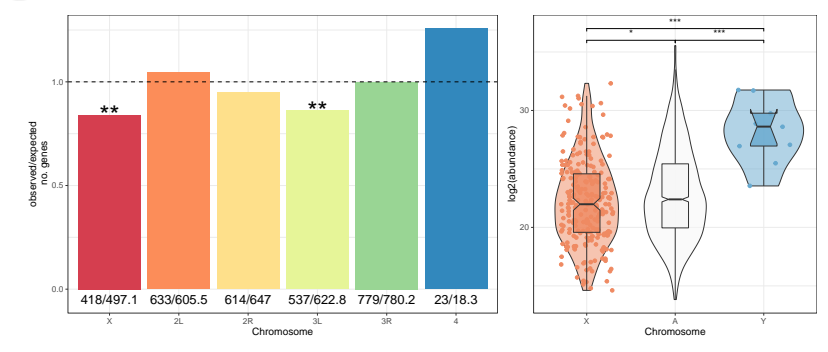

Fig. 4. Chromosomal distribution of DmSP3 proteins. a) Chromosomal distribution of sperm proteins. Numbers below bars are the observed and expected number of genes on each chromosome, respectively, and the dashed line indicates the null expectation. Asterisks represent results from comparing the observed to expected number of genes using the $\chi^{2}$ distribution after multiple testing correction. b) Abundance of sperm proteins found on autosomes ('A') and sex chromosomes (' $X$ ' or ' $Y$ '). Points, representing individual proteins, are omitted from autosomes for clarity. Asterisks represent results from pairwise Wilcoxen rank-sum test corrected for multiple testing using the Benjamini-Hochberg procedure. n.s., non-significant; ${ }^{*} p$ $<0.05 ;{ }^{* \star} p<0.01 ;{ }^{* \star *} p<0.001$.

M. Seminal fluid proteins identified in the DmSP3. Sfps have been extensively studied in Drosophila with over 600 putative Sfps identified to date including 292 that are considered 'high confidence' (42). A surprisingly high number of Sfps were identified in the DmSP3 (122 'high confidence' Sfps; 156 'low confidence/transferred' Sfps; Table S1) (42). 
bioRxiv preprint doi: https://doi.org/10.1101/2022.02.14.480191; this version posted February 14, 2022. The copyright holder for this preprint (which was not certified by peer review) is the author/funder, who has granted bioRxiv a license to display the preprint in perpetuity. It is made available under aCC-BY-NC-ND 4.0 International license.

M Seminal fluid proteins identified in the DmSP3

Table 3. Human disease homologs in the DmSP3. Most common human disease phenotypes from the Online Mendelian Inheritance in Man database (OMIM.org) associated with $D$. melanogaster genes found in the DmSP3. $\mathrm{N}=$ number of $D$. melanogaster genes associated with each phenotype. Similar disease phenotypes (marked with an asterisk) have been grouped. Complete list of disease associations can be found in Table S15.

\begin{tabular}{lc} 
OMIM phenotype & N \\
\hline Autism, Susceptibility To; AUTS20, AUTSX1, AUTSX2 & $27^{*}$ \\
Ciliary Dyskinesia, Primary; CILD40, CILD3, CILD7 & $25^{*}$ \\
Spermatogenic Failure; SPGF39, SPGF45, SPGF46 & $24^{*}$ \\
Myopathy; CFTD, MFM2, Fatal Infantile Hypertonic, Alpha-B Crystallin-Related & $24^{*}$ \\
Hypertension, Essential & 23 \\
Type 2 Diabetes Mellitus; T2D & 21 \\
Asperger Syndrome, X-Linked, Susceptibility To; ASPGX1, ASPGX2 & $18^{*}$ \\
Cataract, Multiple Types; CTRCT16, CTRCT9 & $16^{*}$ \\
Ichthyosis, Congenital, Autosomal Recessive; ARCI4A, ARCI4B & $16^{*}$ \\
46, XY Sex Reversal 8; SRXY8 & 12 \\
Colorectal Cancer; CRC & 11 \\
Encephalopathy, Familial, With Neuroserpin Inclusion Bodies; FENIB & 11 \\
Ghosal Hematodiaphyseal Dysplasia; GHDD & 10 \\
Plasminogen Activator Inhibitor-1 Deficiency & 10 \\
Vitamin D-Dependent Rickets, Type 3; VDDR3 & 10 \\
Deafness, Autosomal Recessive 91; DFNB91 & 9 \\
Leukemia, Acute Myeloid; AML & 9 \\
Maturity-Onset Diabetes of The Young, Type 8, With Exocrine Dysfunction; MODY8 & 9 \\
Pseudoxanthoma Elasticum; PXE & 9 \\
Cardiomyopathy, Dilated, 1II; CMD1II & 8 \\
Charcot-Marie-Tooth Disease, Axonal, Type 2F; CMT2F & 8 \\
Neuronopathy, Distal Hereditary Motor, Type IIB; HMN2B & 8 \\
Surfactant Metabolism Dysfunction, Pulmonary, 3; SMDP3 & 8 \\
\hline
\end{tabular}

Table 4. Y-linked sperm proteins in the DmSP3. Genes are rank ordered by mean abundance and link to male fertility from gene knockout/knockdown experiments (40, 41) are shown.

\begin{tabular}{llcc} 
FBgn & Name & Ranked abundance (\%) & Sterile \\
\hline FBgn0267433 & male fertility factor k15 & 98.8 & Yes \\
FBgn0267432 & male fertility factor k13 & 98.8 & Yes \\
FBgn0058064 & Aldehyde reductase Y & 95.5 & No \\
FBgn0001313 & male fertility factor k12 & 93.6 & Yes \\
FBgn0046323 & Occludin-Related Y & 92.9 & No \\
FBgn0267449 & WD40 Y & 86.4 & Yes \\
FBgn0267592 & Coiled-Coils Y & 86 & Not studied \\
FBgn0046697 & Ppr-Y & 78.3 & No \\
FBgn0046698 & Protein phosphatase 1, Y-linked 2 & 65.2 & No \\
\hline
\end{tabular}
Sfps and the remaining DmSP3 (Kruskal-Wallace rank-sum test, $\chi^{2}=4.28$, df $=1, p=0.118$; Fig. 5a) and 44 'high confidence' Sfps were at, or above, the median abundance of the DmSP3 (Table S5).

We therefore examined the binding characteristics of the Sfps by washing purified sperm with a strong anionic detergent (Triton X-100) known to disrupt plasma membranes. Following detergent treatment 1600 proteins were identified, the majority $(1063 / 1600 ; 66 \%)$ identified by 2 or more unique

${ }_{450}$ peptides. We identified 198 proteins that were lower abundance in PBST compared to controls (Table S6) and three proteins more abundant in PBST samples (Fig. 5b).

Of the 60 'high confidence' Sfps identified by two or more unique peptides in experiment two, 17 (28.4\%) were detected in PBST samples in any replicate), and 29 (48.3\%)

were found at significantly lower abundance in PBST samples, together suggesting these proteins are weakly bound or found on the sperm plasma membrane. The remaining 14 (23.3\%) Sfps showed no significant difference in abundance, suggesting tight association with sperm (Table 5). Additionally, 13 out of $53(24.5 \%)$ RPs detected in experiment two were significantly lower in abundance after PBST treatment. Proteins lower in abundance after PBST treatment showed GO enrichment of multicellular organism reproduction, mitochondrial transport, transmembrane transport, cytoplasmic translation, and sarcomere organisation (BP). Thus, as expected, PBST treatment stripped lipids and membrane- and membrane- bound proteins (including Sfps) from sperm (Table S7).

In experiment three, we washed sperm samples with high molar salt expected to weaken ionic bonds and eliminate non-specific protein binding to sperm (including Sfps). We 

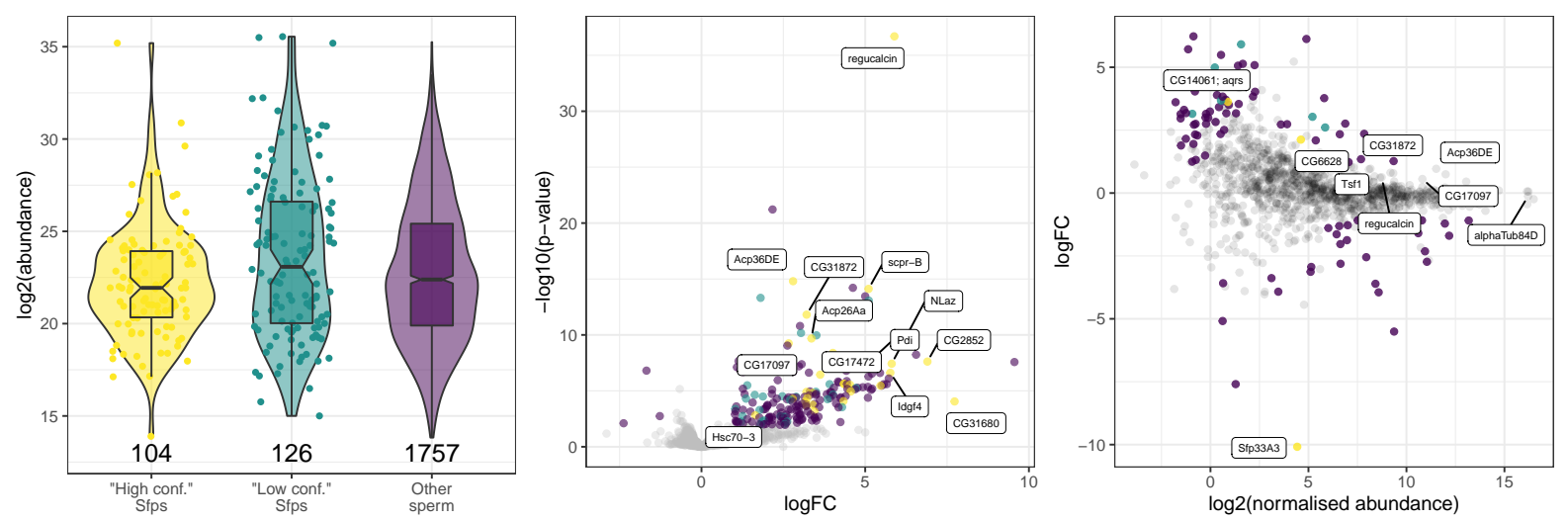

Fig. 5. Seminal fluid proteins in the DmSP3. a) log2 abundance of proteins found in the DmSP3 classified as 'high confidence' Sfps, 'low confidence or transferred' Sfps by Wigby et al. (42), or remaining sperm proteins. Points, representing individual proteins, are omitted from 'other sperm' for clarity. b) Volcano plot for difference between PBST treatment vs. the average of both controls (Halt and NoHalt) in experiment two. Positive values indicate higher abundance in controls. c) MA plot for difference between NaCl treatment vs. PBS control. Positive values indicate higher abundance in $\mathrm{NaCl}$ treatment. For b) and c) points are coloured as in a) denoting 'high confidence' (yellow) and 'low confidence/transferred' (turquoise) Sfps or remaining sperm proteins (purple) that showed significant differences in abundance based on a $\mid$ logFC $\mid>1$ and false discovery rate corrected $p$-value $<0.05$. Several Sfps are labelled in $\mathrm{b}$ ) that showed differential abundance between treatments. Sfps are labelled in $\mathrm{c}$ ) that were among the top $10 \%$ most abundant proteins, and the three Sfps that showed significant differences in abundance between treatments (Sfp33A3, aquarius [CG14061], and CG6628).

identified 1890 proteins, of which $1273(65 \%)$ were identified ods) we performed differtial abundance analysis for 1202 proteins and identified 92 differentially abundant proteins, including 3 Sfps (Sfp33A3, aquarius [CG14061], and CG6628) (Fig. 5c). The remaining 48 'high confidence' Sfps we identified in this experiment did not show significant differential abundance between treatments, with 6 Sfps in the top $20 \%$ most abundance proteins (regucalcin, Acp36DE, CG31872, Transferrin 1, CG17097, and $\alpha$-Tubulin at 84D).

\section{N. Gene - protein abundance concordance. To explore}

ationship between protein abundance and gene expression for the 68 'high confidence' Sfps tightly binding to sperm following detergent or salt treatment ('sperm associated Sfps'; Table S8), we compared gene expression (FPKM; fragments per kilobase of transcript per million mapped reads) for all proteins identified in the DmSP3 between the accessory glands, carcass, ovary, and testis using data retrieved from FlyAtlas2 (43). The average expression

Table 5. Seminal fluid proteins remaining in the sperm proteome after PBST treatment.

\begin{tabular}{llc} 
FBgn & Name & Chrom. \\
\hline FBgn0011694 & Ejaculatory bulb protein II & 2R \\
FBgn0261055 & Seminal fluid protein 26Ad & 2L \\
FBgn0004181 & Ejaculatory bulb protein & 2R \\
FBgn0003885 & $\alpha$-Tubulin at 84D & 3R \\
FBgn0260745 & midline fasciclin & 3R \\
FBgn0036970 & Serpin 77Bc & 3L \\
FBgn0036969 & Serpin 77Bb & 3L \\
FBgn0259975 & Seminal fluid protein 87B & $3 \mathrm{R}$ \\
FBgn0034709 & Secreted Wg-interacting molecule & 2R \\
FBgn0264815 & Phosphodiesterase 1c & 2L \\
FBgn0020414 & Imaginal disc growth factor 3 & 2L \\
FBgn0050104 & Ecto-5'-nucleotidase 2 & 2R \\
FBgn0052203 & Serpin 75F & $3 \mathrm{~L}$ \\
FBgn0003748 & Trehalase & 2R \\
\hline
\end{tabular}

of both sperm associated Sfps and the remaining Sfps identified in the DmSP3 was highest in the accessory glands, while the remaining DmSP3 proteins were most highly expressed in testis (Fig. S4a). However, 7 sperm associated Sfps showed higher expression in the testis than accessory glands (Table S9).

The abundance of proteins in the DmSP3 had the strongest correlation $(\beta)$ and best fit $\left(R^{2}\right)$ in the testis $(\beta=$ $0.460, R^{2}=0.133, p<0.001, \mathrm{n}=1498$ ) (Fig. S4b). Protein abundance of sperm associated Sfps was positively correlated with gene expression in the testis $\left(\beta=0.399, R^{2}=0.152, p\right.$ $=0.006, \mathrm{n}=49)$, but not the accessory glands $(p=0.246)$, carcass $(p=0.052)$, or ovary $(p=0.271)$. The abundance of remaining Sfps identified in the DmSP3 was positively correlated with gene expression in the accessory glands $(\beta=0.274$, $\left.R^{2}=0.197, p=0.004, \mathrm{n}=41\right)$ and testis $\left(\beta=0.281, R^{2}=\right.$ $0.147, p=0.040, \mathrm{n}=29)$, but not the carcass $(p=0.109)$ or ovary $(p=0.677)$ (Fig. S4c). Therefore, our results suggest sperm associated Sfps show tighter regulation with gene expression in the testis than accessory glands.

O. Gene age. A variety of mechanisms drive genomic and protein diversity including gene duplication and retroposition $(6,31)$ resulting in unique, lineage-specific patterns of gene age (44-46). Newly evolved genes frequently acquire testisbiased gene expression (47) and it was therefore of interest to query the gene age landscape of the DmSP3. There were fewer 'recent' ( $\left.\chi^{2}=6.58, \mathrm{df}=1, p=0.026\right)$, 'melanogaster subgroup' ( $\left.\chi^{2}=9.69, \mathrm{df}=1, p=0.009\right)$, and 'Sophophoragroup' ( $\chi^{2}=5.51, \mathrm{df}=1, p=0.032$ ) age genes than expected by chance, indicating genes encoding sperm proteins are underrepresented in more recent evolutionary time (Fig. 6a). We identified 13 genes of recent origin, of which five were located on the X chromosome (Table S10).

P. Sperm evolutionary rates. Genes in the DmSP3 evolve more slowly than the genome average (Mann-Whitney U test, $p<0.001)$. This pattern remains when considering $\mathrm{X}$-linked 


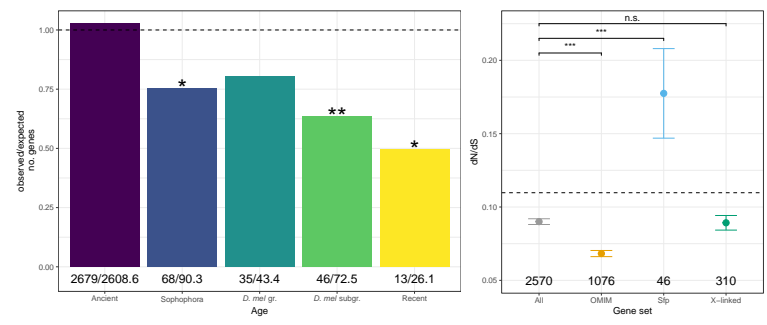

Fig. 6. Sperm evolutionary dynamics. a) Gene age distribution of sperm proteins. Numbers below bars are the observed and expected number of genes in each age class, respectively, and the dashed line at 1 indicates the null expectation. Asterisks represent results from comparing the observed to expected number of genes using the $\chi^{2}$ distribution after multiple testing correction. b) Mean ( \pm standard error) nonsynonymous $(\mathrm{dN})$ to synonymous $(\mathrm{dS})$ nucleotide substitution rate $(\mathrm{dN} / \mathrm{dS})$ estimates for sperm proteins. Asterisks represent results from Mann-Whitney $U$ tests comparing each gene set (OMIM, Sfp, X-linked) to the genome average ("All"), excluding proteins in that set. Dashed line represents the genome average (mean $\mathrm{dN} / \mathrm{dS}=0.110$, standard error $=0.001, \mathrm{n}=11417$ ). Numbers below points indicate numbers of genes in each category. Note: groups are not necessarily mutually exclusive, i.e., 'OMIM' proteins may also be 'X-linked', etc. n.s., non-significant; * $p<$ $0.05 ;{ }^{* *} p<0.01 ;{ }^{* * *} p<0.001$.

sperm proteins compared to the genome average $(p<0.001)$, 530 which evolve at a similar rate to the DmSP3 average ( $p=$ 0.958; Fig. 6b). Sfps in the DmSP3 evolve faster than the DmSP3 average $(p<0.001)$, at a similar rate to other Sfps $(p=0.232$; Fig. S5), whereas genes with a human disease homolog (OMIM.org) evolve more slowly than the DmSP3 average $(p<0.001$; Fig. 6b).

The top 10\%, fastest evolving genes in the DmSP3 $(\mathrm{dN} / \mathrm{dS}$ [mean \pm s.e.] $=0.313 \pm 0.009, \mathrm{n}=257$, Table $\mathrm{S} 11)$ showed GO enrichment for multicellular organism reproduction (BP) and extracellular space (CC) (Table S12). The bot540 tom $10 \%$, slowest evolving genes in the DmSP3 $(\mathrm{dN} / \mathrm{dS}=$ $0.004 \pm 0.0002, \mathrm{n}=258$, Table S13) showed GO BP enrichment for cytoplasmic translation, centrosome duplication, regulation of cell shape, ribosomal large subunit assembly, tricarboxylic acid cycle, ATP hydrolysis coupled proton 545 transport, cell adhesion, oocyte microtubule cytoskeleton polarisation, and endocytosis (Table S14).

\section{Discussion}

In summary, our reanalysis of the D. melanogaster sperm proteome (DmSP3) more than doubled the number of identi-

550 fied proteins, dramatically increased representation of RPs, and highlighted several human neurological disease homologs. LFQ identified highly abundant tubulins, SpermLeucylaminopeptidases (S-Laps), Y-linked sperm proteins and ocnus, a testis-specific protein. LFQ also provided di555 rect evidence for lowered abundances of X-linked sperm proteins. Sperm genes evolve relatively slowly and are underrepresented in recent age classes, consistent with evolutionary constraint acting on the sperm proteome. Finally, we identified a number of Sfps in the DmSP3 which were resistant to 560 detergent or high molar salt treatment, suggesting some are integral to the sperm proteome.

The increased ( $>2$-fold) depth of proteome coverage is likely due to improved protein extraction, efficiency of trypsination/peptide recovery and direct injection methods pre-fractionation has been the method of choice for the analysis of complex proteomes. However, these off-line methods come at a cost: sample loss due to the extra steps involved and the well-known issues of peptide recovery from polyacrylamide gels $(48,49)$. Although work to alleviate this limitation continues to improve this approach, our results suggest that a combination of high SDS concentrations in the initial solubilization and use of immobilized enzymatic digestion using S-Trap technologies greatly enhanced the yield of usable peptides for bottom-up proteomics. The DmSP3 also contained Yolk protein 2 (Yp2), a protein previously found in sperm (50) but undetected in the DmSP1 or DmSP2. As noted by the authors of this study, detection of Yp2 in sperm required large amounts of input protein for detection on immunoblots, suggesting Yp2 was present at very low levels in the testis and sperm (50). Therefore, detection of Yp2 in our study provides additional confidence in the efficacy or our approach.

The sperm proteome is expected to exhibit dynamic gene movement and expression evolution due to its sexspecific expression and essential role for male fertility (6). We found X-linked genes are underrepresented in the sperm proteome, as reported in the DmSP1 (7). Additionally, we show that X-linked sperm proteins were found in significantly lower abundances, consistent with the downstream effects of meiotic sex chromosome inactivation (16-18), and/or resolution of intralocus sexual conflict (51-53). In contrast, more than half of Y-linked proteins (9/16) including known fertility factors were present in the DmSP3 $(40,41)$. Our LFQ analysis revealed all 9 Y-linked protein abundances were above the DmSP average, with 7/9 in the top $10 \%$. This is the first quantitative assessment of this important class of sperm proteins in sperm and adds direct empirical evidence in support of the long-standing hypothesized structural role in the assembly of the sperm axoneme (54).

We found sperm proteins evolve more slowly than the genome average. Slow rates of adaptive evolution could be due to purifying selection or weak selection acting on sperm genes as they are shielded from selection in females $(7,55,56)$. Sperm proteins were also underrepresented in recent evolutionary age classes and over $80 \%$ had human homologs, supporting the idea that sperm genes are under evolutionary constraint. A recent study found Sfps are overrepresented in recent age classes (57), indicating different evolutionary forces acting on sperm vs. non-sperm components of the ejaculate. Sfps in the DmSP3 evolve at a similar rate to Sfps found elsewhere in the genome, and more quickly than the DmSP3 average, suggesting similar evolutionary pressures affecting rates of Sfp evolution across tissue types.

The abundance of RPs in the DmSP3 was unexpected given that sperm are stripped of most cellular machinery prior to maturation. However, sperm may undergo post ejaculatory modifications, perform secondary sexual functions, or provision the developing zygote after fertilization, requiring protein synthesis (58-61). Sperm function beyond delivering a haploid compliment of nuclear material for fertilization still remains relatively underexplored $(59,62,63)$. The presence 
of a large repertoire of core RPs delivered to the egg during fertilization raises the intriguing possibility that paternallyderived ribosomes are active during zygote formation and perhaps beyond.

Another intriguing finding that sperm had higher abundance of RpL22 versus the paralog RpL22-like, opposite from levels found in the testis (36) suggests a complex pattern of paralog switching and selectivity during spermatogenesis. While the functional significance of this selectivity is unknown, they are interesting to consider in the context of the known mRNA repertoire in Drosophila sperm delivered to the egg at fertilization (61). Fully 33\% of the total sperm mRNA repertoire encoded ribosomal proteins (47/142; ref. (61)), a striking coincidence that warrants further study. We also found similarity in the underrepresentation of mitochondrial RPs in both the DmSP3 and brain, providing yet another example of the molecular similarities between these two tissue types (64). Finally, we note that the DmSP3 contains as many as 300 entries with GO annotation terms related to neuronal structure and function, lending additional support to the similarities drawn between the brain and testis.

\section{Q. Possible testis origin of seminal fluid proteins. Al-} though some Sfps were previously identified, but not quantified, in the DmSP2 (14), the unexpectedly high numbers (and in some cases, relative abundances) of Sfps in the DmSP3 adds to an expanding landscape of seminal fluid protein biology. As Sfps are thought to be primarily secreted from the paired accessory glands and the ejaculatory bulb in Drosophila (65), our results raise the possibility that some Sfps are integral to the sperm proteome, are secreted from the testes or seminal vesicles, or bind to sperm prior to mixing in the ejaculatory duct. We identified 122 'high confidence' Sfps (42) in the DmSP3 which is unlikely artefactual given that many Sfps were found in multiple biological replicates and in independent experiments. Denaturing the sperm plasma membrane using detergent stripped most (75\%) Sfps from the sperm proteome, suggesting these Sfps are integral to the sperm plasma membrane or bound to sperm advantageously in the seminal vesicles prior to mixing in the ejaculatory duct. High molar salt had little effect on the composition of the sperm proteome, indicating some Sfps are bound strongly to sperm.

We identified 68 'sperm associated Sfps' that were not depleted by detergent or salt treatment. We suggest several of the 'high-confidence' Sfps identified in the DmSP3 that are highly expressed in the testes (Table S9) should be classified as sperm proteins. In addition, $\alpha$-Tubulin at $84 \mathrm{D}$ (FBgn0003885) is a major constituent of microtubules and involved in sperm axoneme assembly, and therefore likely a sperm protein. Notably, Acp36DE was consistently among the most abundant proteins in our experiments. Acp36DE tightly binds sperm and is essential for efficient sperm storage in the female sperm storage organs $(66,67)$. The possibility that Sfps bind to sperm in the seminal vesicles prior to mixing in the ejaculatory duct should be investigated further. Moreover, the potential for the testes, seminal vesicles, or perhaps even sperm cells, to secrete proteins, including Sfps, requires further investigation.

Finally, the DmSP3 contains over 1200 human disease homologs. The prominence of several neurological diseases (e.g., Primary Ciliary Dyskinesia, susceptibility to autism, encephalopathy, and neuropathy) may be related to the shared functional designs of sperm and neurons, cells of extraordinary axial ratios transmitting biological information over large distances. It will be of great interest to tease out the significance of this subset of neural-related DmSP3 proteins in the context of sperm function and its related reproductive activities and possible relevance for study of human diseases.

R. Conclusion. Our reanalysis of the D. melanogaster sperm proteome using improved separation and detection methods and an updated genome annotation highlights several key features of sperm function and evolution, including the prominence of proteins integral to sperm development (tubulins and S-Laps), the dynamic nature of sex-linked sperm genes, and constraints on sperm proteome evolution. We also show the prevalence of many RPs, despite the expectation that sperm are transcriptionally silent. The parallels in ribosomal protein composition and occurrence of several human neurological disease homologs also lends further support to the functional similarities between sperm and neurons. Finally, we demonstrate that a significant number of seminal fluid proteins are found in the sperm proteome raising the possibility that Sfps mix with sperm in the seminal vesicles, or Sfps may be secreted from the testes, seminal vesicles, or even sperm cells.

S. Data availability. Proteomic data have been deposited to the ProteomeXchange Consortium via the PRIDE partner repository (68) with the identified PDXXXXXXXX. All code and analyses are available on GitHub.

T. Author contributions. TLK and MDG conceived the study. TLK and JS performed dissections and LC-MS experiments. MDG and TLK performed analyses and wrote the manuscript. All authors agreed on the final version of the manuscript.

\section{ACKNOWLEDGEMENTS}

We would like to thank Caitlin McDonough-Goldstein and Maria Vibranovski for helpful discussion, Alison Wright, Daniela Palmer, and Leeban Yusef for advice analysing evolutionary rates, and Eric Sedore and Larne Pekowsky from the Syracuse University HTC Campus Grid and NSF award ACl-1341006 for providing computing services. We are also grateful to the authors whose data was used in this study for making data publicly available and the curators of FlyBase.org for continued maintenance of this essential resource. This work was funded in part by the Biodesign Institute and ASU Knowledge Enterprise Core Research Facilities.

\section{References}

1. Steve Dorus and Timothy L. Karr. Sperm proteomics and genomics. In Sperm Biology: An Evolutionary Perspective, pages 435-469. Academic press, Burlington, MA, first edition, 2009.

2. Helen L. Bayram, Amy J. Claydon, Philip J. Brownridge, Jane L. Hurst, Alan Mileham, Paula Stockley, Robert J. Beynon, and Dean E. Hammond. Cross-species proteomics in analysis of mammalian sperm proteins. Journal of Proteomics, 135:38-50, March 2016. ISSN 18743919. doi: 10.1016/j.jprot.2015.12.027.

3. Melissah Rowe, Sheri Skerget, Matthew A. Rosenow, and Timothy L. Karr. Identification and characterisation of the zebra finch (Taeniopygia guttata) sperm proteome. Journal of Proteomics, October 2018. ISSN 1874-3919. doi: 10.1016/j.jprot.2018.10.009.

4. Scott Pitnick, David J. Hosken, and Timothy R. Birkhead. Sperm morphological diversity. In Sperm Biology: An Evolutionary Perspective, pages 69-149. Academic press, Burlington, MA, first edition, 2009. 
bioRxiv preprint doi: https://doi.org/10.1101/2022.02.14.480191; this version posted February 14, 2022. The copyright holder for this preprint (which was not certified by peer review) is the author/funder, who has granted bioRxiv a license to display the preprint in perpetuity. It is made available under aCC-BY-NC-ND 4.0 International license.

$\mathrm{T}$ Author contributions

5. Ariel F. Kahrl, Rhonda R. Snook, and John L. Fitzpatrick. Fertilization mode drives sperm length evolution across the animal tree of life. Nat Ecol Evol, pages 1-12, June 2021. ISSN 2397-334X. doi: 10.1038/s41559-021-01488-y.

6. Elaine C. Rettie and Steve Dorus. Drosophila sperm proteome evolution. Spermatogenesis, 2(3):213-223, July 2012. ISSN 2156-5554. doi: 10.4161/spmg.21748.

745

7. Steve Dorus, Scott A Busby, Ursula Gerike, Jeffrey Shabanowitz, Donald F Hunt, and Timothy L. Karr. Genomic and functional evolution of the Drosophila melanogaster sperm proteome. Nature Genetics, 38(12):1440-1445, December 2006. ISSN 1061-4036, 15461718. doi: $10.1038 / n g 1915$.

8. Timothy Karr, L. Fruit flies and the sperm proteome. Human Molecular Genetics, 16(R2): R124-R133, July 2007. ISSN 0964-6906, 1460-2083. doi: 10.1093/hmg/ddm252.

9. Jürgen Cox and Matthias Mann. Quantitative, High-Resolution Proteomics for Data-Driven Systems Biology. Annual Review of Biochemistry, 80(1):273-299, 2011. doi: 10.1146/ annurev-biochem-061308-093216.

10. Ruedi Aebersold and Matthias Mann. Mass-spectrometric exploration of proteome structure and function. Nature, 537(7620):347-355, September 2016. ISSN 1476-4687. doi: 10.1038/ nature 19949.

11. Lei Zhao, Xiaoji Cong, Linhui Zhai, Hao Hu, Jun-Yu Xu, Wensi Zhao, Mengdi Zhu, Minjia Tan, and Bang-Ce Ye. Comparative evaluation of label-free quantification strategies. Journal of Proteomics, 215:103669, March 2020. ISSN 1874-3919. doi: 10.1016/j.jprot.2020. 103669.

12. Fengchao Yu, Sarah E. Haynes, and Alexey I. Nesvizhskii. IonQuant Enables Accurate and Sensitive Label-Free Quantification With FDR-Controlled Match-Between-Runs. Molecular \& Cellular Proteomics, 20, January 2021. ISSN 1535-9476, 1535-9484. doi: 10.1016/j. mcpro.2021.100077.

765 13. Timothy L. Karr. Reproductive proteomics comes of age. Molecular \& Cellular Proteomics, 18(Supplement 1):S1-S5, March 2019. ISSN 1535-9476, 1535-9484. doi: 10.1074/mcp. E119.001418.

14. Elizabeth R. Wasbrough, Steve Dorus, Svenja Hester, Julie Howard-Murkin, Kathryn Lilley, Elaine Wilkin, Ashoka Polpitiya, Konstantinos Petritis, and Timothy L. Karr. The Drosophila melanogaster sperm proteome-II (DmSP-II). Journal of Proteomics, 73(11):2171-2185, October 2010. ISSN 1874-3919. doi: 10.1016/j.jprot.2010.09.002.

15. Carine Barreau, Elizabeth Benson, Elin Gudmannsdottir, Fay Newton, and Helen WhiteCooper. Post-meiotic transcription in Drosophila testes. Development, 135(11):1897-1902, June 2008. ISSN 0950-1991. doi: 10.1242/dev.021949.

775 16. Maria D. Vibranovski, Hedibert F. Lopes, Timothy L. Karr, and Manyuan Long. StageSpecific Expression Profiling of Drosophila Spermatogenesis Suggests that Meiotic Sex Chromosome Inactivation Drives Genomic Relocation of Testis-Expressed Genes. PLOS Genetics, 5(11):e1000731, November 2009. ISSN 1553-7404. doi: 10.1371/journal.pgen. 1000731 .

780 17. Maria D. Vibranovski, Domitille S. Chalopin, Hedibert F. Lopes, Manyuan Long, and Timothy L. Karr. Direct Evidence for Postmeiotic Transcription During Drosophila melanogaste Spermatogenesis. Genetics, 186(1):431-433, September 2010. doi: 10.1534/genetics.110. 118919.

18. Sharvani Mahadevaraju, Justin M. Fear, Miriam Akeju, Brian J. Galletta, Mara M. L. S. Pinheiro, Camila C. Avelino, Diogo C. Cabral-de-Mello, Katie Conlon, Stafania Dell'Orso, Zelalem Demere, Kush Mansuria, Carolina A. Mendonça, Octavio M. Palacios-Gimenez, El Ross, Max Savery, Kevin Yu, Harold E. Smith, Vittorio Sartorelli, Haiwang Yang, Nasser M. Rusan, Maria D. Vibranovski, Erika Matunis, and Brian Oliver. Dynamic sex chromosome expression in Drosophila male germ cells. Nature Communications, 12(1):892, February 2021. ISSN 2041-1723. doi: 10.1038/s41467-021-20897-y.

19. Lukas Käll, Jesse D. Canterbury, Jason Weston, William Stafford Noble, and Michael J. MacCoss. Semi-supervised learning for peptide identification from shotgun proteomics datasets. Nat Methods, 4(11):923-925, November 2007. ISSN 1548-7105. doi: 10.1038/nmeth1113.

20. Da Wei Huang, Brad T. Sherman, and Richard A. Lempicki. Systematic and integrative analysis of large gene lists using DAVID bioinformatics resources. Nat Protoc, 4(1):44-57, January 2009. ISSN 1750-2799. doi: 10.1038/nprot.2008.211.

21. Paul Shannon, Andrew Markiel, Owen Ozier, Nitin S. Baliga, Jonathan T. Wang, Danie Ramage, Nada Amin, Benno Schwikowski, and Trey Ideker. Cytoscape: A Software Environment for Integrated Models of Biomolecular Interaction Networks. Genome Res., 13(11): 2498-2504, January 2003. ISSN 1088-9051, 1549-5469. doi: 10.1101/gr.1239303.

22. Gabriela Bindea, Bernhard Mlecnik, Hubert Hackl, Pornpimol Charoentong, Marie Tosolini, Amos Kirilovsky, Wolf-Herman Fridman, Franck Pagès, Zlatko Trajanoski, and Jérôme GaIon. ClueGO: A Cytoscape plug-in to decipher functionally grouped gene ontology and pathway annotation networks. Bioinformatics, 25(8):1091-1093, April 2009. ISSN 1367-4803. doi: 10.1093/bioinformatics/btp101.

23. Alison E. Wright, Peter W. Harrison, Fabian Zimmer, Stephen H. Montgomery, Marie A. Pointer, and Judith E. Mank. Variation in promiscuity and sexual selection drives avian rate of Faster-Z evolution. Molecular Ecology, 24(6):1218-1235, 2015. ISSN 1365-294X. doi: 10.1111/mec.13113.

810 24. Andrew D Yates, Premanand Achuthan, Wasiu Akanni, James Allen, Jamie Allen, Jorge Alvarez-Jarreta, M Ridwan Amode, Irina M Armean, Andrey G Azov, Ruth Bennett, Jyothish Bhai, Konstantinos Billis, Sanjay Boddu, José Carlos Marugán, Carla Cummins, Claire Davidson, Kamalkumar Dodiya, Reham Fatima, Astrid Gall, Carlos Garcia Giron, Lauren Gil, Tiago Grego, Leanne Haggerty, Erin Haskell, Thibaut Hourlier, Osagie G Izuogu, Sophie H Janacek, Thomas Juettemann, Mike Kay, Ilias Lavidas, Tuan Le, Diana Lemos, Jose Gonzalez Martinez, Thomas Maurel, Mark McDowall, Aoife McMahon, Shamika Mohanan, Benjamin Moore, Michael Nuhn, Denye N Oheh, Anne Parker, Andrew Parton, Mateus Patricio, Manoj Pandian Sakthivel, Ahamed Imran Abdul Salam, Bianca M Schmitt, Helen Schuilenburg, Dan Sheppard, Mira Sycheva, Marek Szuba, Kieron Taylor, Anja Thormann, Glen Threadgold, Alessandro Vullo, Brandon Walts, Andrea Winterbottom, Amonida Zadissa, Marc Chakiachvili, Bethany Flint, Adam Frankish, Sarah E Hunt, Garth Ilsley, Myrto Kostadima, Nick Langridge, Jane E Loveland, Fergal J Martin, Joannella Morales, Jonathan M Mudge, Matthieu Muffato, Emily Perry, Magali Ruffier, Stephen J Trevanion, Fiona Cunningham, Kevin L Howe, Daniel R Zerbino, and Paul Flicek. Ensemb 2020. Nucleic Acids Research, 48(D1):D682-D688, January 2020. ISSN 0305-1048. doi: 10.1093/nar/gkz966.

25. Stephen F. Altschul, Warren Gish, Webb Miller, Eugene W. Myers, and David J. Lipman. Basic local alignment search tool. Journal of Molecular Biology, 215(3):403-410, October 1990. ISSN 0022-2836. doi: 10.1016/S0022-2836(05)80360-2.

26. Ari Löytynoja and Nick Goldman. webPRANK: A phylogeny-aware multiple sequence aligner with interactive alignment browser. BMC Bioinformatics, 11(1):579, November 2010. ISSN 1471-2105. doi: 10.1186/1471-2105-11-579.

27. Peter W Harrison, Gregory E Jordan, and Stephen H Montgomery. SWAMP: Sliding Window Alignment Masker for PAML. Evol Bioinform Online, 10:197-204, December 2014. ISSN 1176-9343. doi: 10.4137/EBO.S18193.

28. Ziheng Yang. PAML 4: Phylogenetic Analysis by Maximum Likelihood. Mol Biol Evol, 24(8): 1586-1591, August 2007. ISSN 0737-4038. doi: 10.1093/molbev/msm088.

29. R Core Team. R: A language and environment for statistical computing. R Foundation for Statistical Computing, 2020.

30. Aoife Larkin, Steven J Marygold, Giulia Antonazzo, Helen Attrill, Gilberto dos Santos, Phani V Garapati, Joshua L Goodman, L Sian Gramates, Gillian Millburn, Victor B Strelets, Christopher J Tabone, Jim Thurmond, and FlyBase Consortium. FlyBase: Updates to the Drosophila melanogaster knowledge base. Nucleic Acids Research, 49(D1):D899-D907, January 2021. ISSN 0305-1048. doi: 10.1093/nar/gkaa1026.

31. Yong E. Zhang, Maria D. Vibranovski, Benjamin H. Krinsky, and Manyuan Long. Agedependent chromosomal distribution of male-biased genes in Drosophila. Genome Res, 20(11):1526-1533, November 2010. ISSN 1549-5469. doi: 10.1101/gr.107334.110.

32. Mark D. Robinson, Davis J. McCarthy, and Gordon K. Smyth. edgeR: A Bioconductor package for differential expression analysis of digital gene expression data. Bioinformatics, 26 (1):139-140, January 2010. ISSN 1367-4811. doi: 10.1093/bioinformatics/btp616.

33. Steve Dorus, Elaine C Wilkin, and Timothy L. Karr. Expansion and functional diversification of a leucyl aminopeptidase family that encodes the major protein constituents of Drosophila sperm. BMC Genomics, 12:177, April 2011. ISSN 1471-2164. doi: 10.1186/1471-2164-12-177.

34. John Parsch, Colin D. Meiklejohn, Elisabeth Hauschteck-Jungen, Peter Hunziker, and Daniel L. Hartl. Molecular Evolution of the ocnus and janus Genes in the Drosophila melanogaster Species Subgroup. Molecular Biology and Evolution, 18(5):801-811, May 2001. ISSN 0737-4038. doi: 10.1093/oxfordjournals.molbev.a003862.

35. Shinya Yamamoto, Manish Jaiswal, Wu-Lin Charng, Tomasz Gambin, Ender Karaca, Ghayda Mirzaa, Wojciech Wiszniewski, Hector Sandoval, Nele A. Haelterman, Bo Xiong, Ke Zhang, Vafa Bayat, Gabriela David, Tongchao Li, Kuchuan Chen, Upasana Gala, Tamar Harel, Davut Pehlivan, Samantha Penney, Lisenka E.L.M. Vissers, Joep de Ligt, Shalini N. Jhangiani, Yajing Xie, Stephen H. Tsang, Yesim Parman, Merve Sivaci, Esra Battaloglu, Donna Muzny, Ying-Wooi Wan, Zhandong Liu, Alexander T. Lin-Moore, Robin D. Clark, Cynthia J. Curry, Nichole Link, Karen L. Schulze, Eric Boerwinkle, William B. Dobyns, Rando Allikmets, Richard A. Gibbs, Rui Chen, James R. Lupski, Michael F. Wangler, and Hugo J. Bellen. A Drosophila genetic resource of mutants to study mechanisms underlying human genetic diseases. Cell, 159(1):200-214, September 2014. ISSN 00928674. doi: 10.1016/j.cell.2014.09.002.

36. Tayah Hopes, Karl Norris, Michaela Agapiou, Charley G P McCarthy, Philip A Lewis, Mary J O'Connell, Juan Fontana, and Julie L Aspden. Ribosome heterogeneity in Drosophila melanogaster gonads through paralog-switching. Nucleic Acids Research, (gkab606), July 2021. ISSN 0305-1048. doi: 10.1093/nar/gkab606.

37. Wen Xi Cao, Sarah Kabelitz, Meera Gupta, Eyan Yeung, Sichun Lin, Christiane Rammelt, Christian Ihling, Filip Pekovic, Timothy C. H. Low, Najeeb U. Siddiqui, Matthew H. K. Cheng, Stephane Angers, Craig A. Smibert, Martin Wühr, Elmar Wahle, and Howard D. Lipshitz. Precise Temporal Regulation of Post-transcriptional Repressors Is Required for an Orderly Drosophila Maternal-to-Zygotic Transition. Cell Reports, 31(12), June 2020. ISSN 2211 . 1247. doi: 10.1016/j.celrep.2020.107783.

38. Caitlin E. McDonough-Goldstein, Scott Pitnick, and Steve Dorus. Drosophila oocyte proteome composition covaries with female mating status. Scientific Reports, 11(1):3142, February 2021. ISSN 2045-2322. doi: 10.1038/s41598-021-82801-4.

39. Jiefu Li, Shuo Han, Hongjie Li, Namrata D. Udeshi, Tanya Svinkina, D. R. Mani, Chuanyun Xu, Ricardo Guajardo, Qijing Xie, Tongchao Li, David J. Luginbuhl, Bing Wu, Colleen N. McLaughlin, Anthony Xie, Pornchai Kaewsapsak, Stephen R. Quake, Steven A. Carr, Alice Y. Ting, and Liqun Luo. Cell-Surface Proteomic Profiling in the Fly Brain Uncovers Wiring Regulators. Cell, 180(2):373-386.e15, January 2020. ISSN 0092-8674, 1097-4172. doi: 10.1016/j.cell.2019.12.029.

40. Yassi Hafezi, Samantha R. Sruba, Steven R. Tarrash, Mariana F. Wolfner, and Andrew G. Clark. Dissecting Fertility Functions of Drosophila Y Chromosome Genes with CRISPR. Genetics, 214(4):977-990, April 2020. ISSN 0016-6731, 1943-2631. doi: 10.1534/genetics. 120.302672 .

41. Jiaying Zhang, Junjie Luo, Jieyan Chen, Junbiao Dai, and Craig Montell. The role of $Y$ chromosome genes in male fertility in Drosophila melanogaster. Genetics, 215(3):623633, July 2020. ISSN 1943-2631. doi: 10.1534/genetics.120.303324.

42. Stuart Wigby, Nora C. Brown, Sarah E. Allen, Snigdha Misra, Jessica L. Sitnik, Irem Sepil, Andrew G. Clark, and Mariana F. Wolfner. The Drosophila seminal proteome and its role in postcopulatory sexual selection. Philosophical Transactions of the Royal Society B: Biolog ical Sciences, 375(1813):20200072, December 2020. doi: 10.1098/rstb.2020.0072.

43. David P Leader, Sue A Krause, Aniruddha Pandit, Shireen A Davies, and Julian A T Dow. FlyAtlas 2: A new version of the Drosophila melanogaster expression atlas with RNA-Seq, miRNA-Seq and sex-specific data. Nucleic Acids Research, 46(D1):D809-D815, January 2018. ISSN 0305-1048. doi: 10.1093/nar/gkx976.

44. Henrik Kaessmann, Nicolas Vinckenbosch, and Manyuan Long. RNA-based gene duplication: Mechanistic and evolutionary insights. Nat Rev Genet, 10(1):19-31, January 2009. ISSN 1471-0064. doi: 10.1038/nrg2487.

45. Qianwei Su, Huangyi He, and Qi Zhou. On the Origin and Evolution of Drosophila New Genes during Spermatogenesis. Genes, 12(11):1796, November 2021. ISSN 2073-4425. doi: $10.3390 /$ genes 12111796

46. Manyuan Long, Nicholas W. VanKuren, Sidi Chen, and Maria D. Vibranovski. New Gene Evolution: Little Did We Know. Annu. Rev. Genet., 47(1):307-333, November 2013. ISSN
830 
bioRxiv preprint doi: https://doi.org/10.1101/2022.02.14.480191; this version posted February 14,2022 . The copyright holder for this preprint

(which was not certified by peer review) is the author/funder, who has granted bioRxiv a license to display the preprint in perpetuity. It is made available under aCC-BY-NC-ND 4.0 International license.

0066-4197, 1545-2948. doi: 10.1146/annurev-genet-111212-133301.

47. Esther Betrán, Kevin Thornton, and Manyuan Long. Retroposed New Genes Out of the X in Drosophila. Genome Res., 12(12):1854-1859, January 2002. ISSN 1088-9051, 1549-5469. doi: $10.1101 /$ gr.604902.

48. Ayako Takemori, David S. Butcher, Victoria M. Harman, Philip Brownridge, Keisuke Shima, Daisuke Higo, Jun Ishizaki, Hitoshi Hasegawa, Junpei Suzuki, Masakatsu Yamashita, Joseph A. Loo, Rachel R. Ogorzalek Loo, Robert J. Beynon, Lissa C. Anderson, and Nobuaki Takemori. PEPPI-MS: Polyacrylamide-Gel-Based Prefractionation for Analysis of intat Proteoforms and Protein Complexes by Mass Spectrometry. J. Proteome Res., 19(9): 3779-3791, September 2020. ISSN 1535-3893. doi: 10.1021/acs.jproteome.0c00303.

49. Nobuaki Takemori, Ayako Takemori, Piriya Wongkongkathep, Michael Nshanian, Rachel R. Ogorzalek Loo, Frederik Lermyte, and Joseph A. Loo. Top-down/Bottom-up Mass Spectrometry Workflow Using Dissolvable Polyacrylamide Gels. Anal. Chem., 89(16):82448250, August 2017. ISSN 0003-2700. doi: 10.1021/acs.analchem.7b00357.

50. Magdalena M. Majewska, Agnieszka Suszczynska, Joanna Kotwica-Rolinska, Tomasz Czerwik, Bohdan Paterczyk, Marta A. Polanska, Piotr Bernatowicz, and Piotr Bebas. Yolk proteins in the male reproductive system of the fruit fly Drosophila melanogaster: Spatial and temporal patterns of expression. Insect Biochemistry and Molecular Biology, 47:23-35, April 2014. ISSN 09651748. doi: 10.1016/j.ibmb.2014.02.001.

51. Michael Parisi, Rachel Nuttall, Daniel Naiman, Gerard Bouffard, James Malley, Justen Andrews, Scott Eastman, and Brian Oliver. Paucity of genes on the drosophila X chromosome showing male-biased expression. Science, 299(5607):697-701, January 2003. ISSN 00368075.

52. Richard P. Meisel, John H. Malone, and Andrew G. Clark. Disentangling the relationship between sex-biased gene expression and X-linkage. Genome Res., 22(7):1255-1265, January 2012. ISSN 1088-9051, 1549-5469. doi: 10.1101/gr.132100.111.

53. Qi Zhou and Doris Bachtrog. Sex-specific adaptation drives early sex chromosome evolution in Drosophila. Science, 337(6092):341-345, July 2012. ISSN 0036-8075, 1095-9203.

940 doi: $10.1126 /$ science. 1225385 . melanogaster binds a tektin-like protein. Genetics, 133(3):569-579, March 1993. ISSN 1943-2631. doi: 10.1093/genetics/133.3.569.

55. Amy L. Dapper and Michael J. Wade. Relaxed selection and the rapid evolution of reproductive genes. Trends in Genetics, 0(0), July 2020. ISSN 0168-9525. doi: 10.1016/j.tig. 2020.06.014.

56. Helen M. Southern, Mitchell A. Berger, Philippe G. Young, and Rhonda R. Snook. Sperm morphology and the evolution of intracellular sperm-egg interactions. Ecology and Evolution, 8(10):5047-5058, May 2018. ISSN 2045-7758. doi: 10.1002/ece3.4027.

57. Bahar Patlar, Vivek Jayaswal, José M. Ranz, and Alberto Civetta. Non-adaptive molecular evolution of seminal fluid proteins in Drosophila. Evolution, n/a(n/a), 2021. ISSN 1558-5646. doi: 10.1111/evo.14297.

58. Yael Gur and Haim Breitbart. Mammalian sperm translate nuclear-encoded proteins by mitochondrial-type ribosomes. Genes Dev, 20(4):411-416, February 2006. ISSN 08909369. doi: 10.1101/gad.367606.

59. Timothy L. Karr, William J. Swanson, and Rhonda R. Snook. The evolutionary significance of variation in sperm-egg interactions. In Tim R. Birkhead, David J. Hosken, and Scott Pitnick, editors, Sperm Biology, pages 305-365. Academic Press, London, January 2009. ISBN 978-0-12-372568-4. doi: 10.1016/B978-0-12-372568-4.00008-2.

60. Scott Pitnick, Mariana F. Wolfner, and Steve Dorus. Post-ejaculatory modifications to sperm (PEMS). Biological Reviews, 95(2):365-392, 2020. ISSN 1469-185X. doi: 10.1111/brv. 12569.

61. Bettina E. Fischer, Elizabeth Wasbrough, Lisa A. Meadows, Owen Randlet, Steve Dorus, Timothy L. Karr, and Steven Russell. Conserved properties of Drosophila and human spermatozoal mRNA repertoires. Proc. R. Soc. B., 279(1738):2636-2644, July 2012. ISSN 0962-8452, 1471-2954. doi: 10.1098/rspb.2012.0153.

62. Simone Immler. The sperm factor: Paternal impact beyond genes. Heredity, 121(3):239247, September 2018. ISSN 1365-2540. doi: 10.1038/s41437-018-0111-0.

63. Stephen A. Krawetz. Paternal contribution: New insights and future challenges. Nat Rev Genet, 6(8):633-642, August 2005. ISSN 1471-0064. doi: 10.1038/nrg1654.

64. Bárbara Matos, Stephen J. Publicover, Luis Filipe C. Castro, Pedro J. Esteves, and Margarida Fardilha. Brain and testis: More alike than previously thought? Open Biology, 11(6): 200322, 2021. doi: 10.1098/rsob.200322.

65. Frank W. Avila, Laura K. Sirot, Brooke A. LaFlamme, C. Dustin Rubinstein, and Mariana F. Wolfner. Insect seminal fluid proteins: Identification and function. Annu. Rev. Entomol., 56 (1):21-40, 2011. ISSN 0066-4170. doi: 10.1146/annurev-ento-120709-144823.

66. D M Neubaum and M F Wolfner. Mated Drosophila melanogaster females require a seminal fluid protein, Acp36DE, to store sperm efficiently. Genetics, 153(2):845-857, October 1999. ISSN 0016-6731.

67. Frank W. Avila and Mariana F. Wolfner. Cleavage of the Drosophila seminal protein Acp36DE in mated females enhances its sperm storage activity. Journal of Insect Physiology, 101:66-72, August 2017. ISSN 0022-1910. doi: 10.1016/j.jinsphys.2017.06.015.

68. Yasset Perez-Riverol, Jingwen Bai, Chakradhar Bandla, David García-Seisdedos, Suresh Hewapathirana, Selvakumar Kamatchinathan, Deepti J Kundu, Ananth Prakash, Anika Frericks-Zipper, Martin Eisenacher, Mathias Walzer, Shengbo Wang, Alvis Brazma, and Juan Antonio Vizcaíno. The PRIDE database resources in 2022: A hub for mass spectrometry-based proteomics evidences. Nucleic Acids Research, 50(D1):D543-D552, January 2022. ISSN 0305-1048. doi: 10.1093/nar/gkab1038. 
bioRxiv preprint doi: https://doi.org/10.1101/2022.02 14.480191; this version posted February 14, 2022. The copyright holder for this preprint (which was not certified by peer review) is the author/funder, who has granted bioRxiv a license to display the preprint in perpetuity. It is made available under aCC-BY-NC-ND 4.0 International license.

$\mathrm{T}$ Author contributions

\section{Supplementary Note 1: Supplementary information}
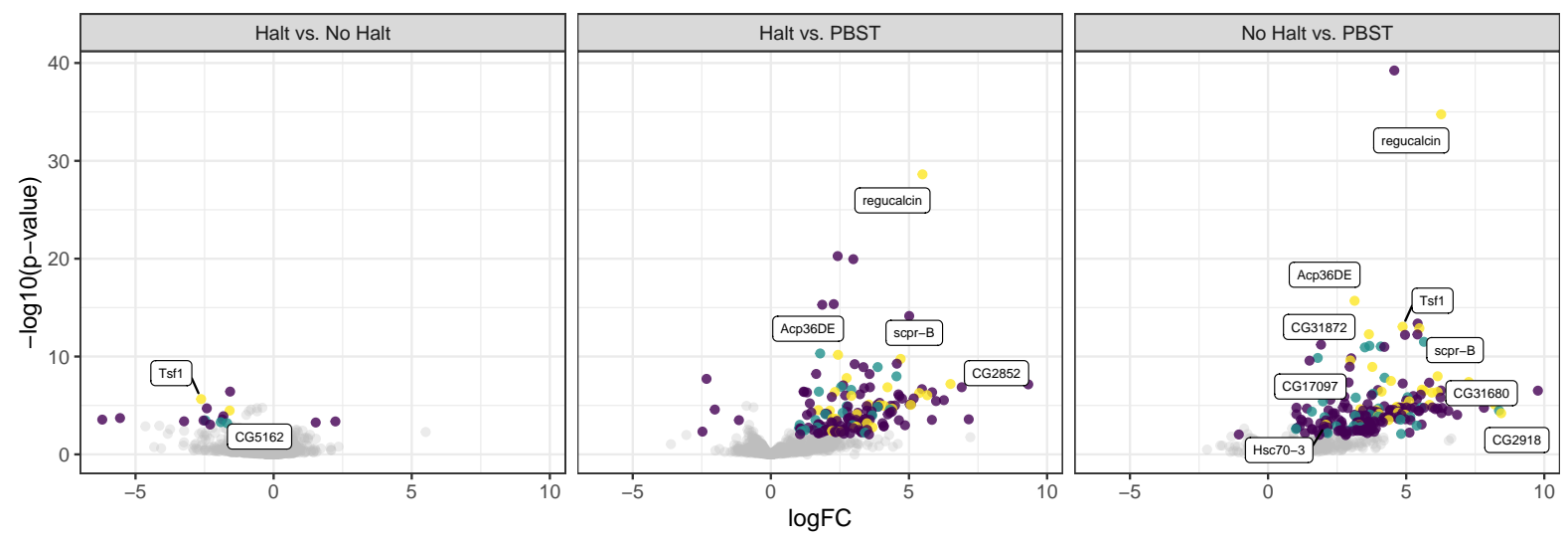

SFP.high • SFP.low • Sperm.only

Fig. S1. Volcano plots from pairwise analyses between treatments in experiment two, denoting 'high confidence' (yellow) and 'low confidence/transferred' (turquoise) Sfps or remaining sperm proteins (purple) that showed significant differences in abundance based on a $|\log F C|>1$ and false discovery rate corrected $p$-value $<0.05$. Several Sfps are labelled that showed differential abundance between treatments.

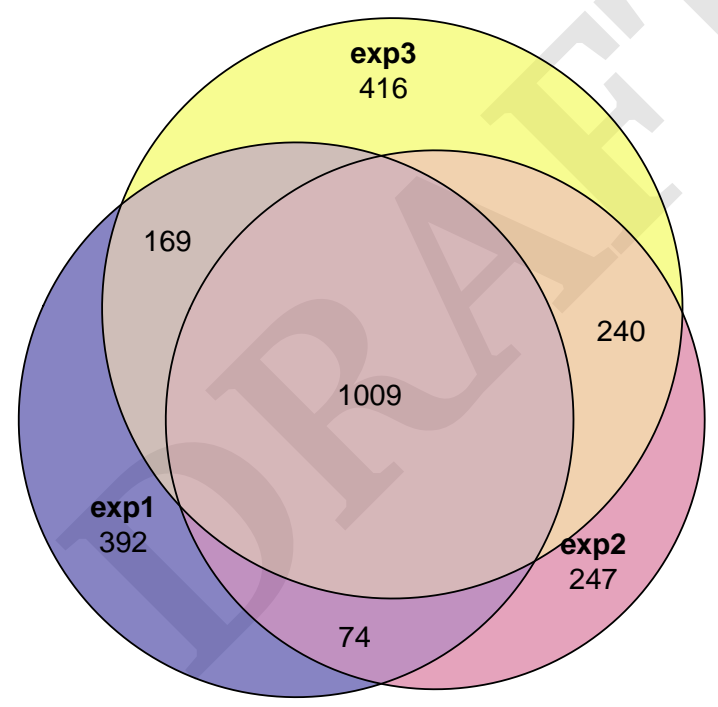

Fig. S2. Overlap between proteins identified in each experiment in the current study. 
bioRxiv preprint doi: https://doi.org/10.1101/2022.02.14.480191; this version posted February 14,2022 . The copyright holder for this preprint (which was not certified by peer review) is the author/funder, who has granted bioRxiv a license to display the preprint in perpetuity. It is made available under aCC-BY-NC-ND 4.0 International license.
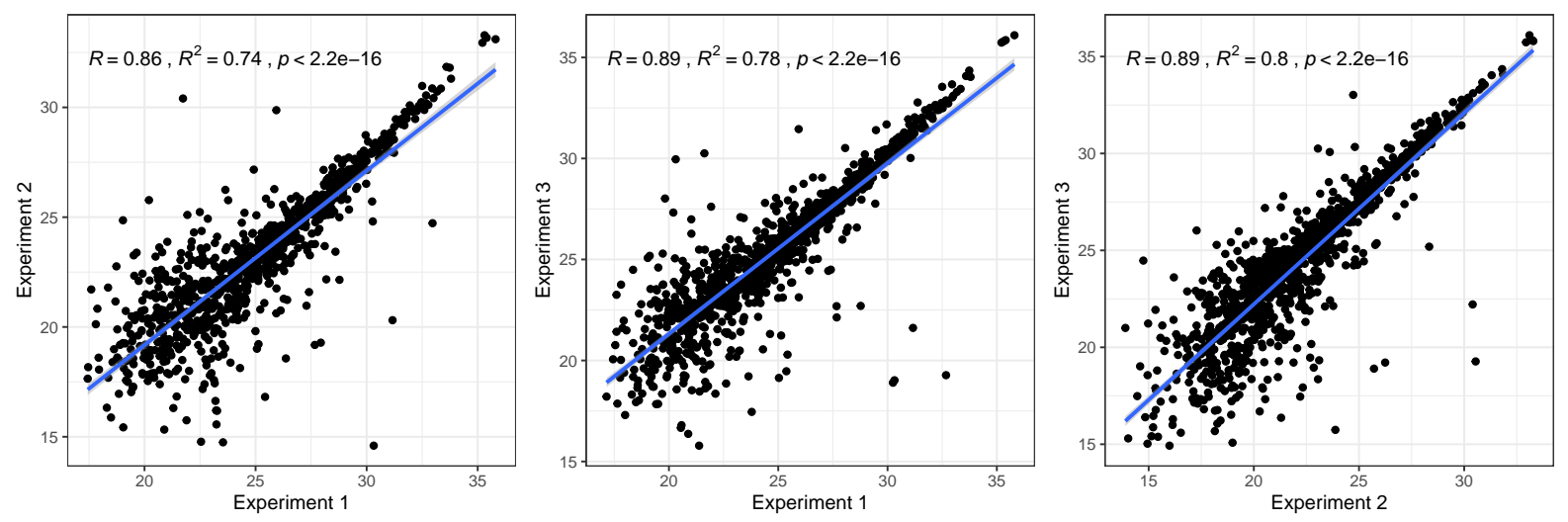

Fig. S3. Correlations in average protein abundance between each experiment in the current study. Mean protein abundance was calculated across all replicates for each experiment, except experiment two which excluded the PBST treatment. Shown are Pearson's correlations and line of best fit.
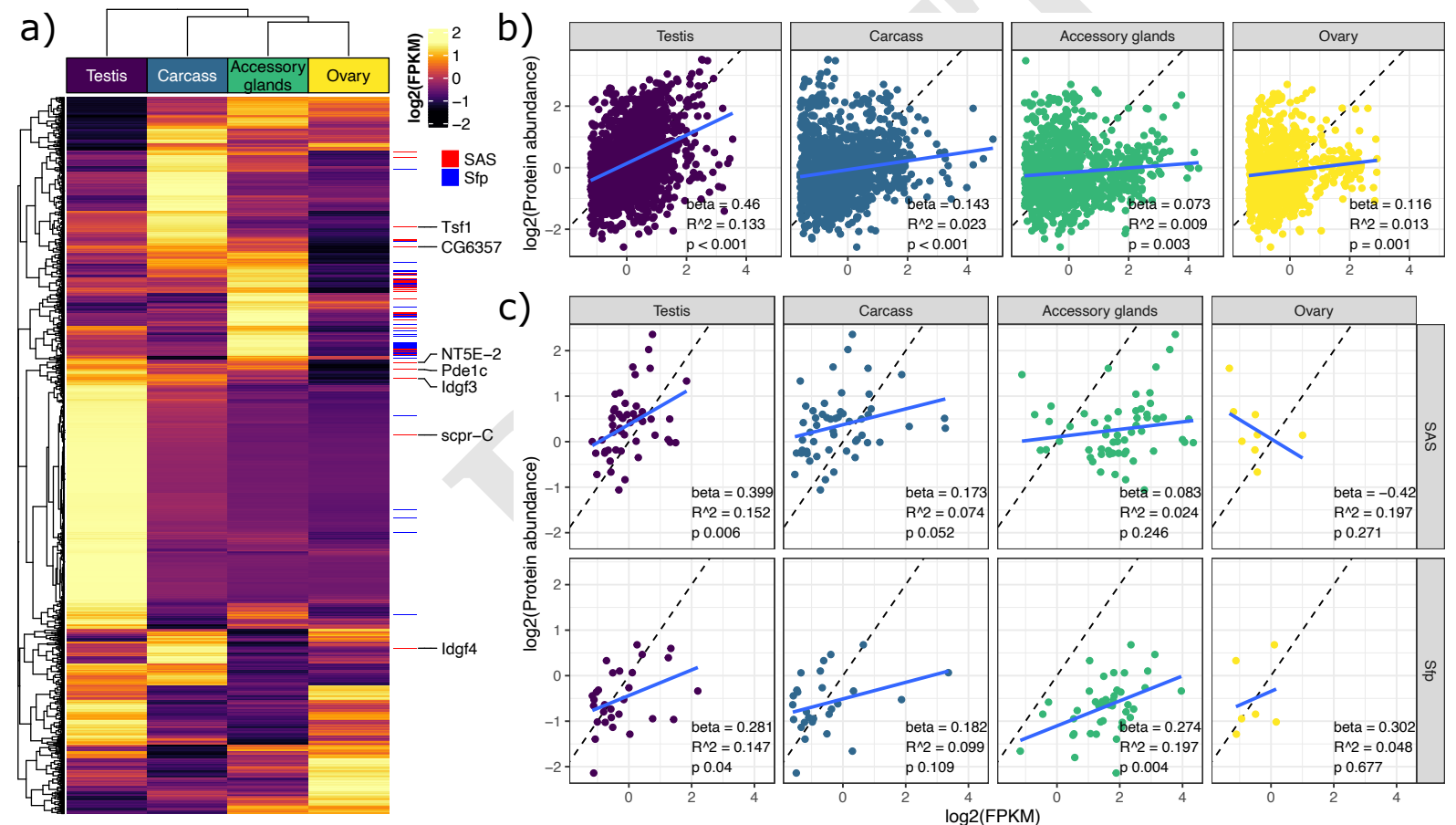

c)
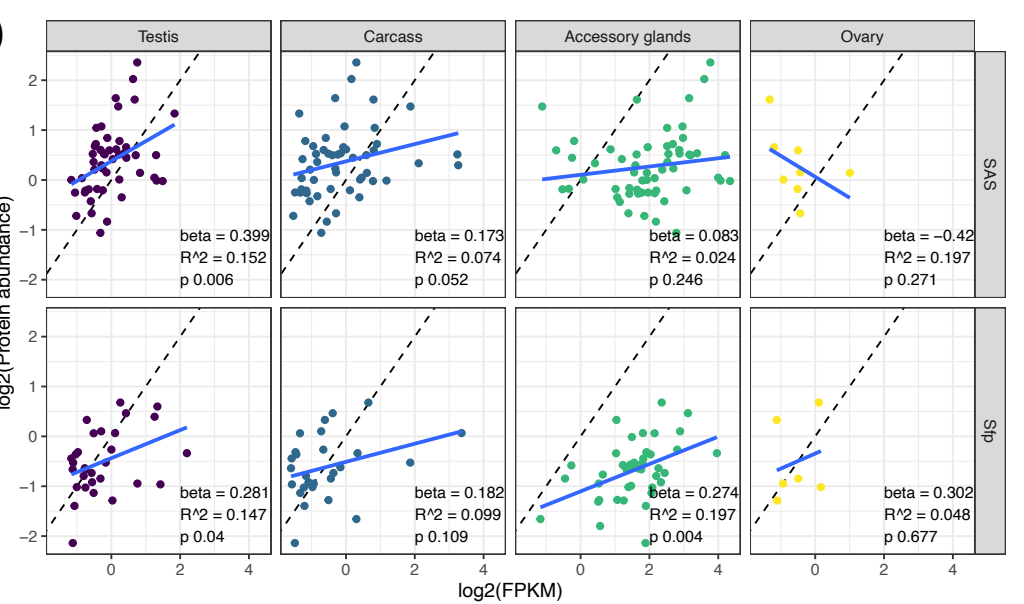

Fig. S4. Gene - protein abundance concordance in the DmSP3. a) Heatmap of mRNA expression of DmSP3 genes $(n=2673)$ in the accessory glands, carcass, ovary, and testis. Data retrieved from from FlyAtlas2 (43) are log2(FPKM) scaled per gene. The 7 'high confidence' Sfps with higher expression in the testis than accessory glands are highlighted on the right. Labels on the right also show 'sperm associated Sfps' (red) and other 'high confidence' Sfps (blue) identified in the DmSP3. b) Linear regressions of gene expression on protein abundance in the testis $(n=1498)$, carcass $(n=1165)$, accessory glands $(n=1001)$, and ovary $(n=825)$. c) Linear regressions of gene expression on protein abundance for 'sperm associated Sfps' (SAS) and remaining Sfps identified in the DmSP in each tissue. b) and c) are linear regressions using z-score log2-transformed values after filtering genes with log2-FPKM < 2. Blue lines are model fits from a linear regression, dashed lines indicate a perfect correlation between gene expression and protein abundance. 
bioRxiv preprint doi: https://doi.org/10.1101/2022.02.14.480191. this version posted February 14, 2022. The copyright holder for this preprint (which was not certified by peer review) is the author/funder, who has granted bioRxiv a license to display the preprint in perpetuity. It is made available under aCC-BY-NC-ND 4.0 International license.

$\mathrm{T}$ Author contributions

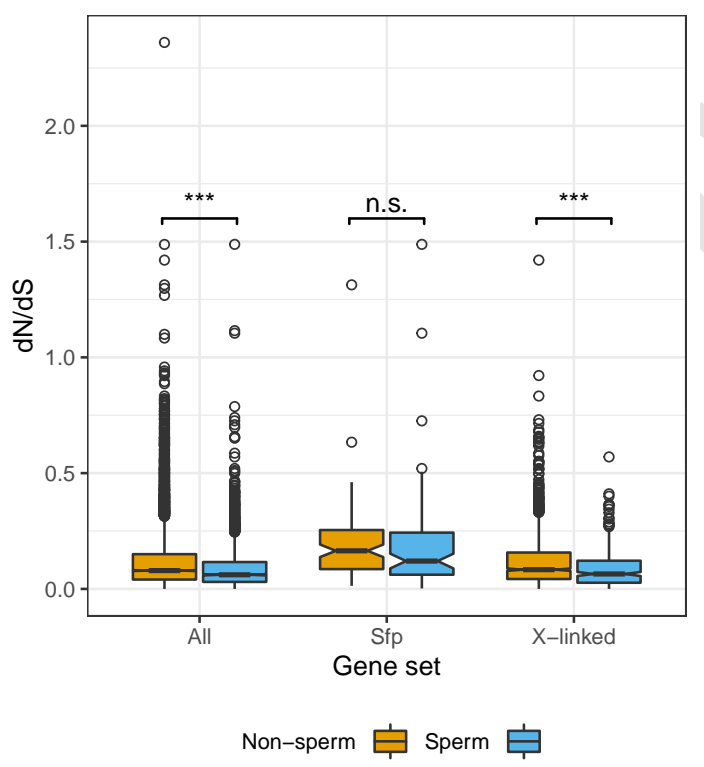

Fig. S5. Nonsynonymous $(\mathrm{dN})$ to synonymous $(\mathrm{dS})$ nucleotide substitution rate $(\mathrm{dN} / \mathrm{dS})$ estimates for proteins in the DmSP3 or elsewhere. Asterisks represent results from Mann-Whitney $U$ tests; n.s., non-significant; ${ }^{* \star *}, p<0.001$. 